\title{
Polymorphic centromere locations in the pathogenic yeast Candida parapsilosis
}

Mihaela Ola', Caoimhe E. O'Brien ${ }^{1}$, Aisling Y. Coughlan², Qinxi Ma ${ }^{1}$, Paul D. Donovan ${ }^{1}$, Kenneth H. Wolfe ${ }^{2}$ and Geraldine Butler ${ }^{1 *}$.

${ }^{1}$ School of Biomolecular and Biomedical Science and ${ }^{2}$ School of Medicine, Conway Institute, University College Dublin, Belfield, Dublin 4, Ireland.

*Corresponding author

Geraldine Butler, School of Biomolecular and Biomedical Science, Conway Institute, University College Dublin, Belfield, Dublin 4, Ireland.

Email: gbutler@ucd.ie

Phone+353-1-7166885 
1 ABSTRACT

3 Centromeres pose an evolutionary paradox: strongly conserved in function, but rapidly

4 changing in sequence and structure. However, in the absence of damage, centromere

5 locations are usually conserved within a species. We report here that isolates of the

6 pathogenic yeast species Candida parapsilosis exhibit within-species polymorphism for the

7 location of centromeres on two of its eight chromosomes. Its old centromeres have an

8 inverted-repeat (IR) structure, whereas its new centromeres have no obvious structural

9 features, but are located within $30 \mathrm{~kb}$ of the old site. Centromeres can therefore move

10 naturally from one chromosomal site to another, apparently spontaneously and in the absence of any significant changes in DNA sequence. Our observations are consistent with or long IRs, or the ability to form cruciforms. We also find that centromeres have been hotspots for genomic rearrangements in the $C$. parapsilosis clade.

\section{INTRODUCTION}

Centromeres are the point of assembly of the kinetochore, the position at which the spindle microtubules are connected to the chromosomes, enabling efficient and accurate separation of chromosome/chromatid pairs during cell division. Most eukaryotes have large "regional" centromeres that have been proposed to be epigenetically determined. They are specified by arrays of chromatin, compacted by di- or tri-methylation at lysine 9 of histone $\mathrm{H} 3$ (H3K9me2/3). The position of the centromere in most species is determined by the presence of a variant of histone $\mathrm{H} 3$, called CENP-A in mammals, or Cse4 in yeast.

Centromere repositioning occurs on an evolutionary time scale, leading to the formation of evolutionarily new centromeres (ENCs). ENCs have played an important role in speciation, including in many mammals (Rocchi et al. 2012; Stanyon et al. 2008). An ancient ENC at one chromosome in orangutans is polymorphic; individuals can be homozygous for either the old or the new centromere, or heterozygous for both (Locke et al. 2011; Rocchi et al. 2012). The new centromere location lacks the repetitive alpha satellites observed at other centromeres. In addition, damage to, or loss of, existing centromeres can be rescued by the formation of new (neo) centromeres at different locations. Neocentromere formation following damage has been observed in human clinical samples, as well as in other primates, in equidae, marsupials, plants and yeasts (reviewed in (Rocchi et al. 2012;

36 Schubert 2018; Burrack and Berman 2012)). Movement of centromeres among individuals 37 within a species in a non-clinical context is much more rarely described. A small number of 
38

39

40

41

42

43

44

neocentromeres formed in human cells that have no obvious clinical effect have been reported; these were usually observed during routine amniocentesis (reviewed in (Rocchi et al. 2012)). In addition, the location of one centromere in the horse (devoid of satellite DNA) varies among individuals (Purgato et al. 2015; Wade et al. 2009). The mechanisms underlying the formation of new centromeres are not fully understood, though many are likely to be associated with chromosomal inversion and translocation (Schubert 2018). The formation of neocentromeres following damage is particularly well studied in the yeast Candida albicans (Burrack and Berman 2012). Koren et al (Koren et al. 2010) suggested that in this species, centromeres are associated with the presence of early origins of replication, and that the formation of neocentromeres changes the activity of nearby origins.

Basic centromere organization is conserved in many fungi, including the basidiomycetes and the filamentous ascomycetes (Friedman and Freitag 2017). Centromeres in the budding yeasts (the Saccharomycotina) have undergone substantial changes associated with the loss of the lysine methylation machinery (Malik and Henikoff 2009). Within Saccharomycotina, the Saccharomycetaceae clade, containing the model yeast Saccharomyces cerevisiae, is by far the best studied. These species have small "point" centromeres, where function is determined by sequence. The $S$. cerevisiae centromere consists of three conserved regions called Centromere Determining Elements - CDEI, CDEII and CDEIII (Schulman and Bloom 1991). Cse4 is present in one nucleosome at the centromere (Meluh et al. 1998; Furuyama and Biggins 2007; Henikoff and Henikoff 2012). Similar point centromeres are found in other Saccharomycetaceae species (Kitada et al. 1997; Gordon et al. 2011; Mattei et al. 2002). In Naumovozyma species, the sequences of the CDE elements are different, but they still act as point centromeres (Kobayashi et al. 2015). The point centromeres in S. cerevisiae are among the fastest evolving sequences in the genome (Bensasson et al. 2008). However, point centromeres are not present in most fungal genomes (Malik and Henikoff 2009).

Centromere structure has also been investigated in other families in the Saccharomycotina, including the Pichiaceae and the CUG-Ser1 clade. Within the Pichiaceae, centromere structure is known in Kuraishia capsulata and Komagataella phaffii. In K. capsulata, centromeres lie in 2-6 kb regions with low GC content, and a 200 bp motif is conserved across some chromosomes (Morales et al. 2013). In K. phaffii the centromeres consist of a 1 $\mathrm{kb}$ central (mid) region, flanked by a $2 \mathrm{~kb}$ inverted repeat (IR) (Coughlan et al. 2016). There is no conservation in sequence among the four centromeres in K. phaffii, and Cse4 localizes across the mid region and the IR. 
75 The CUG-Ser1 clade within the Saccharomycotina contains many Candida and other

76 species, characterized by translating CUG as serine rather than leucine (Ohama et al. 1993).

77 The centromeres of Candida albicans and Candida dubliniensis are described as "small

78 regional"; they are characterized by gene-free regions of 4-18 kb, with 3-5 kb occupied by

79 Cse4 (Sanyal et al. 2004; Roy and Sanyal 2011; Padmanabhan et al. 2008). The flanking

80 compact chromatin extends up to $25 \mathrm{~kb}$ for $C$. albicans CEN7 (centromere of chromosome

81 7) (Sreekumar et al. 2019). There is no sequence conservation between centromeres of

82 different chromosomes. There are short unique inverted repeats surrounding C. albicans

83 CEN1, CEN4, and CENR, and longer repeats surrounding CEN5 (Sanyal et al. 2004). In the

84 related species Candida tropicalis, the centromere cores are all flanked by IRs, and there is

85 significant sequence conservation between different centromeres (Chatterjee et al. 2016).

86 Centromeres in the more distantly related Clavispora lusitaniae have $4 \mathrm{~kb}$ regions occupied

87 by Cse4, with no sequence conservation (Kapoor et al. 2015). The C. lusitaniae centromeres

88 lie in regions with low GC content, which has also been proposed to mark centromeres in the

89 CUG-Ser1 clade species Debaryomyces hansenii and Scheffersomyces stipitis (Lynch et al.

90 2010). The putative centromeres in these latter species contain clusters of retrotransposons

91 (Lynch et al. 2010; Coughlan et al. 2016).

92

93 In this work, we experimentally determined the location of centromeres in the CUG-Ser1

94 clade yeast, Candida parapsilosis, and we inferred the locations in the related species $C$.

95 orthopsilosis and C. metapsilosis. We identified C. parapsilosis centromeres by ChIP-Seq

96 and we show that they usually have an IR structure. However, we also identified one

97 C. parapsilosis isolate in which two centromeres have moved, indicating that centromere

98 location in this species is polymorphic. The new centromere locations are $<30 \mathrm{~kb}$ from the

99 old ones, but they do not have any IR structure. These are the first examples of the birth of

100 centromeres de novo in natural fungal isolates, and among the few observed in any species

101 (Purgato et al. 2015; Wade et al. 2009; Locke et al. 2011; Rocchi et al. 2012). The new

102 centromeres have no obvious structure, and we conclude that centromeres can change

103 easily from IR-type to apparently epigenetic-type over short time scales, as well as over

104 evolutionary time. In addition, we find that chromosomal rearrangements between $C$.

105 parapsilosis and its closest relatives are enriched at centromeres, and that multiple

106 rearrangements have occurred at, or very close to the centromeres, indicating fragility and

107 dynamic evolution. 


\section{Identification of centromeres in C. parapsilosis}

113

Many fungal centromeres are located in large intergenic regions and may be flanked by IR sequences. When we looked for regions that matched these criteria in the genome of $C$. parapsilosis CDC317 (the sequenced reference genome (Butler et al. 2009)) we identified one candidate centromere per chromosome (Fig. 1A,B). These regions range from 5.8 to 7.1 $\mathrm{kb}$ and lack genes. Each contains an inverted repeat (IR) sequence (shown in red in the dot matrix plot Fig. 1A), flanking a middle (mid) sequence. The IRs vary in size. Some are relatively short (e.g. 443 bp on chromosome 6) and in others the repeat region is broken into several sections (e.g. chromosome 1, total size $\sim 1600 \mathrm{bp}$ ). The similarity between IRs ranges from 85 to $96.7 \%$. Importantly, the sequence of the IRs are conserved among chromosomes, and the conservation extends beyond the IRs (Fig. 1B, black boxes). All IRs are predicted to form large secondary structures using RNAFold (Lorenz et al. 2011). However, there is no conservation among the mid regions that lie between the IRs on different chromosomes.

To validate these predictions, we determined the location of the variant histone $\mathrm{H} 3, \mathrm{Cse} 4$, by chromatin immunoprecipitation (ChIP). C. parapsilosis has a diploid genome. We introduced three copies of a 9 amino acid epitope from human influenza hemagglutinin (HA), near the $N$ terminus of both Cse4 alleles using CRISPR-Cas9 editing together with a synthetic repair template (Lombardi et al. 2017) (Fig. 1C). The epitope was introduced into Cse4 twice independently in two different strains $-C$. parapsilosis CLIB214, which is the type strain, and C. parapsilosis 90-137, originally isolated from orbital tissue (Tavanti et al. 2005) and which can be efficiently edited using CRISPR-Cas9 (Lombardi et al. 2017, Lombardi et al. 2019a). We confirmed that the tagged protein is expressed and that it does not interfere with growth of the tagged strains, and we used ChIP-PCR to show that Cse4 binding is enriched at the predicted CEN1 sequence (Fig. S1).

To identify all the regions in the genome where Cse4 binds, we combined chromatin immunoprecipitation with DNA sequencing (ChIP-seq). We obtained one very strong ChIPseq signal per chromosome that was present in only the immunoprecipitated Cse4-HA strains, and not in the input chromatin (Fig. 1D). We also identified a signal from the ribosomal DNA on chromosome 7 , an artifact due to the high copy number which is also present in the control sample. More detailed analysis shows that the Cse4 signals from $C$. parapsilosis CLIB214 correspond with the regions that were bioinformatically identified as centromeres (Fig. 2). The centromeres are in regions that are devoid of open reading frames, and are generally low in transcription (Fig. 2). Unlike C. tropicalis (Chatterjee et al. 
149 2016) but similar to K. phaffii (Coughlan et al. 2016) Cse4 binding extends beyond the mid

150 regions into the IRs, reducing in frequency toward the ends of the repeats.

\section{Polymorphic centromere locations in C. parapsilosis}

The Cse4 signal in C. parapsilosis $90-137$ is very similar to C. parapsilosis CLIB214 (Fig. (Fig. 2). However, there are surprising differences at CEN1 and CEN5. For chromosome 1, there is a signal at the expected centromere in $C$. parapsilosis $90-137$, similar to $C$. parapsilosis CLIB214. However, there is an additional signal, approximately $17 \mathrm{~kb}$ away in 90-137 (Fig. 2). This second signal, or neocentromere, partially overlaps two open reading frames, CPAR2_101630 and CPAR2_101640, which are transcribed in C. parapsilosis CLIB214 (RNA track in Fig. 2). The difference is even more striking on chromosome 5. Here, C. parapsilosis 90-137 has no obvious Cse4 signal at the expected position of CEN5 (the small number of reads shown is an artefact of the mapping process, resulting from the presence of repeat sequences). Instead, the Cse4 signal is localized approximately $29 \mathrm{~kb}$ away, again overlapping transcribed ORFs, CPAR2_502960 and CPAR2_502970. There are no IRs surrounding the new centromeres, and there is no sequence relationship with other centromeric regions.

We considered that the occurrence of neocentromeres in C. parapsilosis 90-137 might coincide with possible rearrangements of the chromosomes in this isolate. We therefore determined the genome structure of the Cse4-HA tagged strain using long read sequencing (Oxford Nanopore). The nuclear genome was assembled into 12 scaffolds $>1 \mathrm{~kb}$ in size (Fig. 3). The assembly failed at CEN6 on scaffolds 9/10. However, Fig. 3 shows that chromosomes 1 and 5 are collinear between C. parapsilosis 90-137/Cse4-HA and the reference genome, including around the centromere regions. The IR structures and mid region at the original CEN1 and CEN5 locations are intact in $C$. parapsilosis 90-137, and are 98 - 99\% identical to the reference genome. chromosomes. The centromere relocations are associated with a transition from a structured (IR) format to a format with no obvious structure or sequence dependence, within a single species. On chromosome 5 , it is likely that the centromeres on both copies of this chromosome have moved to a new location. It is possible that $C$. parapsilosis $90-137$ is

184 heterozygous at CEN1, with Cse4 at the expected location on one copy of chromosome 1

185 and at a new location on the other copy. 
Genomic rearrangements in C. orthopsilosis coincide with centromere locations

C. parapsilosis is closely related to $C$. orthopsilosis and C. metapsilosis; they are all members of the $C$. parapsilosis sensu lato clade (Tavanti et al. 2005). We surmised that the centromeres in these other species may have a similar structure to $C$. parapsilosis. The $C$. orthopsilosis 90-125 reference assembly (Riccombeni et al. 2012; Schröder et al. 2016) is not fully assembled at putative centromeres, so we used a minION assembly of this strain from Lombardi et al (Lombardi et al. 2019b). We identified one large region per chromosome likely to represent the centromere. The size of the regions ranges from $4.9 \mathrm{~kb}$ to $7.1 \mathrm{~kb}$ (Fig. 4A). Candidates on chromosomes 1,2, 5, 6 and 7 have a similar structure to $C$. parapsilosis centromeres. A pair of IR sequences, varying in size from 788 bp on chromosome 5 to 2.2 $\mathrm{kb}$ on chromosome 6 , flank a core region of $\sim 3 \mathrm{~kb}$. The similarity between IRs ranges from 91.0 to $99.8 \%$, the sequences are conserved among chromosomes, and for chromosomes 5,6 , and 7 the conservation among chromosomes extends beyond the IRs. The remaining inferred centromeres (CEN3, 4, 8) do not contain IR sequences. However, 135 bp to $2.2 \mathrm{~kb}$ of the flanking regions surrounding the $2.6-3.4 \mathrm{~kb}$ mid regions are conserved with other centromeres. Like in C. parapsilosis, there is no conservation between the mid regions (not just the IR-less ones) share significant sequence similarity with any of the $C$. parapsilosis CEN regions.

207

We compared the conservation of centromere position and gene order between $C$. parapsilosis and $C$. orthopsilosis using SynChro, a tool designed to visualize synteny blocks in eukaryotic genomes (Drillon et al. 2014). Putative orthologs between the two species were assigned by identifying Reciprocal Best Hits (RBHs). Fig. 4B shows the locations of genes in C. orthopsilosis that have a $\mathrm{RBH}$ in C. parapsilosis. Each chromosome is assigned a specific color. Fig. 4C shows the locations of the same RBHs on the C. parapsilosis chromosomes, colored with respect to $C$. orthopsilosis chromosomes. It is immediately obvious that there is

215 strong conservation of synteny between $C$. orthopsilosis and $C$. parapsilosis, as we have 216 described previously (Riccombeni et al. 2012). One chromosome pair (chromosome 7 in 217 each species) is essentially collinear, as shown by the brown color (Fig. 4B,C). Most of the 218 other chromosomes are represented by two major colors in C. parapsilosis, indicating that 219 there has been one major translocation per chromosome between $C$. parapsilosis and $C$. 220 orthopsilosis. 
222 Overlaying the position of the mapped centromeres shows that most of the evolutionary rearrangements between $C$. parapsilosis and $C$. orthopsilosis involve breakpoints at or near the $C$. parapsilosis centromeres (Fig. $4 \mathrm{C}$ ). For some chromosomes there is a single breakpoint (e.g. chromosome 1). For others, whereas most of the two arms of the $C$. parapsilosis chromosome matches two $C$. orthopsilosis chromosomes, the junction near the centromere includes short sections from a third chromosome (e.g. on chromosome 8). These relationships are explored in Fig. 5, which shows the gene order around each $C$. parapsilosis centromere in more detail. Individual RBHs (identified and visualized using SynChro (Drillon et al. 2014)) are shown. Each C. parapsilosis centromere is compared to all C. orthopsilosis chromosomes, and syntenic blocks are highlighted.

Multiple rearrangements have occurred exactly at, or very close to, the centromere on almost all chromosomes (Fig. 5). For example, on C. parapsilosis chromosome 1, genes to the right of the centromere are syntenic with genes on $C$. orthopsilosis chromosome 2 , and genes to the left of the centromere are syntenic with $C$. orthopsilosis chromosome 6 (Fig. $5 A)$. The break in synteny coincides exactly with the location of the predicted centromeres on the two $C$. orthopsilosis chromosomes and with $C$. parapsilosis CEN1. More complex rearrangements are seen at CEN2, CEN4, CEN6, and CEN8 (Fig. 5). In each of these examples, there is a break in synteny at the $C$. parapsilosis centromere, so that the left and right flanks of the $C$. parapsilosis centromeres match two different $C$. orthopsilosis chromosomes, and the breakpoints in $C$. orthopsilosis also occur at or near its centromeres. However, in these four cases there are also additional rearrangements nearby, which at CEN2 (Fig. 5B) corresponds with a third centromere in C. orthopsilosis on chromosome 4.

Even on chromosome 7 (Fig. 5G), which is almost collinear between the two species, there has been an inversion beside the centromere. C. parapsilosis CEN3 is also collinear with $C$. orthopsilosis CEN5 (Fig. 5C). However, there have been two rearrangements on the left of C. parapsilosis CEN3, where a short block of genes on C. parapsilosis chromosome 3 matches a region on $C$. orthopsilosis chromosome 1 . Most of the remainder of the left side of C. parapsilosis chromosome 3 is syntenic with C. orthopsilosis chromosome 6. Something similar is seen at $C$. parapsilosis chromosome 5 (Fig. 5E), except here one rearrangement occurs at a second $C$. orthopsilosis centromere (CEN2). In summary, $C$. parapsilosis has synteny breakpoints relative to $C$. orthopsilosis at 7 of its 8 centromeres, and most of these breakpoints also map to $C$. orthopsilosis centromeres. We examined the sequences around each interchromosomal rearrangement site but did not find any sequence repeats that could have facilitated the rearrangements. 


\section{Genomic rearrangements in C. metapsilosis and L. elongisporus}

C. metapsilosis originated from hybridization between two related species, generating a hybrid with a highly heterozygous diploid genome (Pryszcz et al. 2015). The best assembly of its genome is derived from Illumina sequencing only and is a consensus built from both haplotypes from two different isolates (Pryszcz et al. 2015). Of the nine largest $C$. metapsilosis scaffolds, we identified putative centromeres on seven (Fig. 4D,E). Scaffold 2

266 contained two candidate regions. Closer examination revealed that this scaffold contains a region (around CMET_4044) that is syntenic with two telomeres in C. parapsilosis (chromosomes 5 and 6). We do not know if this represents a recent telomere-to-telomere fusion in C. metapsilosis or if it is an assembly error. We split scaffold 2 at CMET_4044, generating scaffolds $2 \mathrm{~A}$ and $2 \mathrm{~B}$ (Fig. 4E), giving a total of eight centromeres. All the centromeres are surrounded by IRs, which have high levels of sequence similarity among chromosomes. The IRs on scaffolds 5, 6, 7 and 9 are relatively long (2.1-2.6 kb). IRs in conserved regions on scaffolds 6 and 7 are fragmented (Fig. 4D). IRs on scaffolds 1, 2A, 2B, and 3 are highly repetitive, with regions that sometimes overlap. The mid regions of $C$. metapsilosis centromeres vary in size from 1.2 to $2.2 \mathrm{~kb}$, and unlike $C$. parapsilosis and C.orthopsilosis, there is sequence conservation among chromosomes. CEN2B, 5, 6, 7 and 9 share $>75 \%$ identity, and CEN1 is approximately $60 \%$ identical to these (Fig. 4D, Supplemental_Fig. S2).

279

Fig. 4F shows a pattern of interspecies chromosomal breakage at centromeres between $C$. metapsilosis and C. parapsilosis, similar to that seen with $C$. orthopsilosis, although the rearrangements are different and have therefore occurred independently. C. parapsilosis chromosome 6 and C. metapsilosis scaffold 2B are collinear. Most other chromosomes have undergone a major rearrangement at points that correspond to the centromeres of both species. There have been complex rearrangements at these sites, similar to the $C$. orthopsilosis/C. parapsilosis comparisons. For example, the region around C. parapsilosis CEN2 is syntenic with regions near $C$. metapsilosis CEN2A, CEN5 and CEN7. Other apparent rearrangements may reflect gaps in the $C$. metapsilosis assembly (for example $C$. metapsilosis scaffold 8 , which does not contain a centromere, maps to the end of $C$. parapsilosis chromosome 8).

Lodderomyces elongisporus is an outgroup to the $C$. parapsilosis sensu lato species group (Fitzpatrick et al. 2006). We did not find any structures similar to the $C$. parapsilosis centromeres in the L. elongisporus genome (Butler et al. 2009). However, Koren et al (Koren et al. 2010) hypothesised that centromeres in L. elongisporus are adjacent to early-firing 
296

297

298

299

300

301

302

303

304

305

306

307

308

309

310

311

312

313

314

315

316

317

318

319

320

321

322

323

324

325

326

327

328

329

330

331

332

origins of replication, as in $C$. albicans. They identified putative regions by characterizing GC skew, which switches between strands at replication origins. Koren et al (Koren et al. 2010) identified nine candidate centromeres in the 11 largest $L$. elongisporus scaffolds, that lie within intergenic regions and have a strong GC skew. Three may not represent true centromeres; one (on scaffold 9) is adjacent to the rDNA locus (Donovan et al. 2016) and two are in strongly transcribed regions (scaffold 7, scaffold 10) (Donovan et al. 2016) that are probably incorrectly annotated in the L. elongisporus genome. The most likely centromeres and a comparison of the synteny of $C$. parapsilosis with $L$. elongisporus are shown in Supplemental_Figure_S3. There are more rearrangements than observed between C. parapsilosis and C. orthopsilosis or C. metapsilosis. However, C. parapsilosis chromosome 6 and L. elongisporus chromosome 7 are collinear, and major rearrangements in the other chromosomes coincide with the location of the centromeres in C. parapsilosis and several of the remaining centromeres in L. elongisporus (Supplemental_Fig._S3). It is therefore likely that six of the proposed centromere locations in L. elongisporus are correct, and that centromeres are fragile sites in all four species. However, centromere structure in $L$. elongisporus is very different to the $C$. parapsilosis sensu lato species. There are no IRs, and the sequences are mostly unique (Koren et al. 2010). They are therefore more similar to the epigenetic centromeres described in C. albicans and C. dubliniensis (Thakur and Sanyal 2013; Sanyal et al. 2004; Padmanabhan et al. 2008).

In order to identify the number of translocations that have occurred during the evolution of the $C$. parapsilosis clade, we inferred the most likely ancestral chromosomal structure using AnChro (Vakirlis et al. 2016) (Supplemental_Fig_S4). Some of the reference assemblies are quite fragmented, and the number of predicted chromosomes in the ancestral species are probably overestimated (13-15, Supplemental_Fig_S4). It is therefore difficult to fully resolve every rearrangement. However, the synteny comparisons identified 13 interchromosomal breaks between $C$. parapsilosis and C. orthopsilosis, and all are at or close to the centromeres as shown in Fig 5. Most rearrangements occurred on the branch leading to $C$. orthopsilosis (Supplemental_Fig_S4). It is therefore clear that interchromosomal breaks are enriched at centromeres.

\section{DISCUSSION}

Centromeres evolve remarkably rapidly, considering their conserved function (Henikoff et al. 2001). Species in the CUG-Ser1 clade have a very wide range of centromere types (Fig. 6). Centromeres of $C$. albicans and $C$. dubliniensis have been proposed to be epigenetically 
333 determined, and have little obvious sequence similarity, and few IRs (Sanyal et al. 2004;

334 Padmanabhan et al. 2008). We have shown that the centromeres in the $C$. parapsilosis

335 sensu lato species group consist of a mid region that is mostly unique, and is usually

336 surrounded by IR sequences. The centromere structures in the $C$. parapsilosis sensu lato

337 clade are most similar to those of $C$. tropicalis (Fig. 6; (Chatterjee et al. 2016; Padmanabhan

338 et al. 2008)). However, in C. tropicalis, the mid regions of all centromeres are similar ( 80\%

339 identity), and the IRs are highly homogenised. Chatterjee et al (Chatterjee et al. 2016)

340 suggested that the ancestral centromere in Candida species consisted of an IR surrounding

341 a core, and that most of the IRs have been lost in C. albicans and C. dubliniensis. Orthology

342 of the centromeres on each chromosome within the CUG-Ser1 clade, despite their structural

343 variation, is supported by evidence that gene order is partially conserved around

344 centromeres among C. albicans, C. dubliniensis and C. tropicalis (Padmanabhan et al. 2008;

345 Chatterjee et al. 2016). Synteny is conserved between C. albicans CEN3 and C. parapsilosis

346 CEN5, and there is partial conservation of synteny around $C$. albicans CEN5 with

347 centromeres in C. parapsilosis, S. stipitis and C. lusitaniae, even though centromeres do not

348 contain IRs in the latter two species (Lynch et al. 2010; Chatterjee et al. 2016).

349

350 The IR structure of centromeres is likely to be old, because it is also found in some species

351 in the sister clade, the family Pichiaceae (Fig. 6). In Pichia kudriavzevii, the IRs at each CEN

352 are very similar and they are conserved across centromeres. In addition, these IRs share

353 some similarity with mid sequences on other chromosomes (Douglass et al. 2018). In

354 Komagataella phaffii (Pichia pastoris) both the IRs and the mid regions are unique at each

355 CEN (Coughlan et al. 2016). The ancestor of the Pichiaceae and the CUG-Ser1 clade

356 species therefore likely had an IR surrounding a mid region, with unique sequences at each

357 centromere. The IRs have undergone homogenization in several species ( $P$. kudriavzevii, $C$.

358 tropicalis and $C$. parapsilosis sensu lato), and the mid regions have been homogenized in $C$.

359 tropicalis and to a lesser extent in C. metapsilosis. IRs have probably been lost in $C$.

360 albicans, $C$. lusitaniae and $K$. capsulata. In other species in the CUG-Ser1 clade ( $D$.

361 hansenii, S. stipitis) and in the Pichiaceae (Ogataea polymorpha) the CENs are associated

362 with retrotransposons (Ty5-like elements). A retrotransposon (member of the Ty3/Gypsy

363 family) is found at CEN7 in C. tropicalis, C. albicans and C. dubliniensis (Padmanabhan et

364 al. 2008; Chatterjee et al. 2016). DDE-type transposases are found adjacent to $C$.

365 orthopsilosis CEN4 and CEN8, but these are likely to be DNA transposons (Nesmelova and

366 Hackett 2010), more similar to CEN-associated transposons in the basidiomycete

367 Cryptococcus neoformans (Janbon et al. 2014). 
369 It is not clear what the ancestral centromere structure was in the subphylum

370 Saccharomycotina because centromeres have been characterized in very few species

371 outside the Pichiaceae and the CUG-Ser1 clade (Fig. 6). The point centromeres in the

372 Saccharomycetaceae are unusual, and probably represent a derived state (Lefrançois et al.

373 2013; Malik and Henikoff 2009; Kobayashi et al. 2015). Centromeric regions have been

374 identified in Yarrowia lipolytica, an outgroup to the three clades (Fig. 6). These lie in regions

375 of poor GC-content, adjacent to autonomously replicating sequences (Lynch et al. 2010;

376 Fournier et al. 1993). Y. lipolytica centromeres may be small, and have conserved short

377 palindromic repeats of 17 to $21 \mathrm{bp}$ (Yamane et al. 2008). However, the exact structure of the

378 centromere and the location of CENP-A (Cse4) in Y. lipolytica has never been determined.

379 More experimental analysis of centromeres from other clades of the Saccharomycotina is

380 therefore required before conclusions can be drawn about the ancestral centromere

381 structure.

382

383 Kasinathan and Henikoff (Kasinathan and Henikoff 2018) postulated that all centromeres,

384 whether apparently epigenetic or sequence-dependent, share a common feature - they are

385 at regions that can make non-B form DNA. This can be achieved via dyad symmetry (IRs) in

386 the DNA, or by the activity of specific DNA-binding proteins (such as binding of Cbf1 in the

387 Saccharomycetaceae). IRs have the capacity to form cruciform structures, especially when

388 associated with replication origins (Pearson et al. 1996). In particular, Kasinathan and

389 Henikoff (Kasinathan and Henikoff 2018) found that neocentromeres in vertebrates are

390 particularly enriched in regions of short dyad symmetry.

391

392 The formation of "rescue" neocentromeres when the endogenous centromere is damaged

393 has been well studied in C. albicans (reviewed in (Burrack and Berman 2012)). When CEN5

394 or CEN7 is damaged, neocentromeres form, either adjacent to the original centromere or up

395 to $450 \mathrm{~kb}$ away (Ketel et al. 2009; Thakur and Sanyal 2013). Koren et al (Koren et al. 2010)

396 found that natural $C$. albicans $C E N$ s are near early firing replication origins, and that the

397 formation of neocentromeres changes the timing of firing at adjacent origins. By

398 characterizing the switches in base composition skew that occur at replication origins, they

399 predicted that CENs are also near early firing origins of replication in L. elongisporus, $C$.

400 Iusitaniae, and Y. lipolytica (experimentally confirmed by Fournier et al (Fournier et al. 1993)

401 for Y. lipolytica).

402

403 Examination of the known and predicted centromeres in CUG-Ser1 clade species shows that

404 they all contain IRs (either long or short, including retrotransposon LTRs), and/or they are

405 located near early firing replication origins (known or predicted). All of these structures can 
406 form cruciforms, which may be necessary to recruit Cse4, as has been reported for

407 Schizosaccharomyces pombe (Folco et al. 2008). The loss of the IRs at centromeres in $L$.

408 elongisporus, C. Iusitaniae, and from some centromeres in C. albicans and C. dubliniensis,

409 may be compensated by the presence of a nearby early-firing replication origin (Fig. 6).

410 Therefore, there may be no true "epigenetic" centromeres in this clade; as Kasinathan and

411 Henikoff (Kasinathan and Henikoff 2018) suggest, at least some part of centromere

412 formation always requires cruciform or non-B form DNA, however it is made. The

413 neocentromeres formed in C. parapsilosis 90-137 do not contain large IRs like the originals

414 in this species. The hypothesis predicts that the neocentromeres form in regions capable of

415 making cruciform structures, which may be facilitated by transcription. The $C$. parapsilosis

416 neocentromeres are formed at regions that are transcribed, and transcription is known to

417 facilitate centromere activity in S. cerevisiae (Ohkuni and Kitagawa 2011).

418

419 We found that the majority of chromosomal rearrangements between species in the

420 C. parapsilosis/L. elongisporus clade involve breakpoints at or near centromeres, and that in

421 several cases multiple closely-spaced breaks occurred near centromeres. Rearrangements

422 between $C$. albicans and $C$. tropicalis also appear to be enriched around centromeres, which

423 Chaterjee et al. (Chatterjee et al. 2016) suggested was facilitated by repeat sequences.

424 However, rearrangements at centromeres in other species are unusual, and for example

425 were rarely seen in Saccharomycetaceae species (Gordon et al. 2011; Dujon et al. 2004;

426 Vakirlis et al. 2016). It therefore appears that centromeres are hotspots for chromosome

427 breakage in the CUG-Ser1 clade, and particularly in species closely related to $C$.

428 parapsilosis (for example, CENs in C. albicans and C. dubliniensis are collinear

429 (Padmanabhan et al. 2008)). Although fragility may be associated with the presence of

430 repeats (IRs) at the centromeres, and with the similarity of centromere sequences among

431 chromosomes, even the centromeres of $L$. elongisporus, which have no IRs or other repeats,

432 coincide with evolutionary breakpoints (Supplemental_Fig._S3). Interspecies

433 rearrangements of the karyotype by breakage at centromeres has also been reported in the

434 basidiomycete yeast Cryptococcus (Sun et al. 2017).

435

436 There are many unanswered questions about how and why the centromere relocations in

437 C. parapsilosis 90-137 occurred. We do not know how frequent centromere location

438 polymorphism is in C. parapsilosis, but the fact that we observed it in one of only two strains

439 tested, affecting two of eight chromosomes, suggests that it is not rare. Such centromere

440 sliding may also be frequent in other organisms (including humans), but has not been

441 observed because of a lack of investigation (Rocchi et al. 2012). We also do not know what

442 factors caused the original centromere sites to become disused in C. parapsilosis 90-137. 
443 The IR structure at the original sites appears to be intact, so it is unclear why neither allele of

444 CEN5 binds Cse4. Similarly, we do not know what makes the new centromere sites, at both

445 CEN1 and CEN5, attractive for Cse4 binding. They have no repeats and no obvious features

446 such as strong base composition skew. However, they are both within $30 \mathrm{~kb}$ of the original

447 site, which means that diploids heterozygous for Cse4 bound at old and new sites (like at

448 CEN1 in C. parapsilosis 90-137) can still establish proper spindle tension. Similar

449 heterozygous centromeric sites have been reported in orangutans (Locke et al. 2011),

450 horses (Purgato et al. 2015; Wade et al. 2009), and in C. albicans following damage at one

451 allele (Thakur and Sanyal 2013). Lastly, we do not know why the new sites only bind Cse4 in

452 C. parapsilosis 90-137 and not in C. parapsilosis CLIB214. Our discovery of "natural"

453 neocentromeres in C. parapsilosis is one of the few known examples of within-species

454 polymorphism for CEN locations, and provides an ideal opportunity for further future

455 investigation of how centromere location and function is determined (Wade et al. 2009;

456 Locke et al. 2011; Rocchi et al. 2012).

457

458

\section{METHODS}

459

460 Bioinformatic prediction of centromere location. Genomic sequences of intergenic

461 regions larger than $2 \mathrm{~kb}$ were extracted from the reference sequence of $C$. parapsilosis

462 CDC317 (Butler et al. 2009), C. orthopsilosis 90-125 (Riccombeni et al. 2012; Schröder et al.

463 2016), and the chimeric reference assembly of $C$. metapsilosis strains PL429 (SZMC1548)

464 and SZMC8094 (Pryszcz et al. 2015) using a custom script

465 (https://doi.org/10.6084/m9.figshare.9750638.v1). Sequences were compared using Blastn v

466 2.2.26 with default parameters and tabular alignment output (Altschul 1990). An IR pair was

467 defined as a sequence identity $>75 \%$ with a region in the opposite orientation (E-value cutoff

468 0.005). Candidate regions were selected for manual investigation. Predicted centromere

469 locations in the C. orthopsilosis 90-125 reference assembly (Riccombeni et al. 2012;

470 Schröder et al. 2016), available at CGOB (Fitzpatrick et al. 2010), had long regions of

471 ambiguous bases, so we extracted equivalent regions from a minION assembly from

472 Lombardi et al (Lombardi et al. 2019b) (Supplemental_Table_S2). Dot matrix plots were

473 constructed using DNAMAN (www.lynnon.com) with a criterion of 23 matches per $25 \mathrm{bp}$

474 window. Synteny was visualized using SynChro with a delta value of 2 (Drillon et al. 2014),

475 using genome assemblies and annotations from CGOB (Fitzpatrick et al. 2010; Maguire et

476 al. 2013). To reconstruct ancestral genomes, SynChro was run using delta values between 1

477 and 6. The ancestor of $C$. parapsilosis and $C$. orthopsilosis (A1) was reconstructed using

478 AnChro (Vakirlis et al. 2016), varying delta values from 1 to 6 for each branch. $C$.

479 metapsilosis and L. elongisporus were used as outgroups. The best A1 candidate, with the 
480 smallest number of chromosomes (13) and conflicts (6), was chosen as recommended by

481 Vakirlis et al (Vakirlis et al. 2016) (Supplemental_Fig_S4). The A1 reconstruction was then

482 compared to the other genomes using SynChro (delta values 1-6), and a second ancestral

483 genome (A2) was constructed from A1 and C. metapsilosis, with L. elongisporus as an

484 outgroup. The best A2 candidate, with the smallest number of chromosomes (15) and

485 conflicts (1) and the highest number of genes (4409) was chosen (Supplemental_Fig_S4).

486 Interchromosomal breaks were identified using pairwise comparison of synteny maps.

487

488 Tagging Cse4. C. parapsilosis strains CLIB214 and 90-137 were edited using a tRNA

489 plasmid based CRISPR-Cas9 gene editing system as described by Lombardi et al.

490 (Lombardi et al. 2017, 2019a). Primers gRNA_CSE4_TOP and gRNA_CSE4_BOT were

491 annealed and cloned into pCP-tRNA and $5 \mu \mathrm{g}$ plasmid was transformed together with $5 \mu \mathrm{g}$

492 of a 594 bp synthetic DNA fragment containing a section of the $\mathrm{H} 3$ histone variant Cse4 with

493 a 3xHA (hemagglutinin) tag inserted between amino acids 69 and 70, and 250bp homology

494 arms (Integrated DNA Technologies USA, Supplemental_Fig._S1). Transformants were

495 selected on YPD agar supplemented with $200 \mu \mathrm{g} / \mathrm{ml}$ nourseothricin and screened by colony

496 PCR using primers CSE4_N_RT_fw and CSE4_col_inTag_rv. Two clones were sequenced

497 by Sanger sequencing (MWG/Eurofins). Loss of pCP-tRNA was induced by patching

498 transformants onto YPD agar without nourseothricin. For Western blots, protein extracts

499 were prepared from $15 \mathrm{~A}_{600}$ units of $C$. parapsilosis 90-137 and two Cse4-HA tagged strains

500 cultured overnight in YPD. Cell pellets were washed in $500 \mu \mathrm{l}$ water, re-suspended in $500 \mu \mathrm{l}$

501 ice-cold extraction buffer (1x PBS, 0.1\% Tween 20, 1mM PMFS) and homogenized with

502 glass beads. The protein extract was separated by centrifugation at $10000 \mathrm{rpm}$ at $4 \square .20 \mu \mathrm{l}$

503 protein extracts diluted 1:1 (v/v) with ice-cold 2x Laemmli sample buffer (Sigma-Aldrich)

504 were separated by $12 \%$ SDS-PAGE, at $200 \mathrm{~V}$ constant voltage for $1 \mathrm{~h}$, and electro-blotted

505 onto nitrocellulose membranes at $100 \mathrm{~V}$ for $45 \mathrm{~min}$. Immunoblotting was performed using the

506 mouse epitope tag antibody, Anti-HA.11 (BioLegend), at a 1:1000 dilution in milk/TBS

507 blocking buffer (5 g non-fat dry milk to $100 \mathrm{ml}$ TBS - $100 \mathrm{mM}$ Tris- $\mathrm{HCl}, \mathrm{pH}$ 7.5, $150 \mathrm{mM}$

$508 \mathrm{NaCl}$ ) and HRP-conjugated secondary antibody Anti-mouse IgG (Cell Signaling Technology)

509 at 1:2000 dilution. Immunoblots were detected using the Pierce ECL Western Blotting

510 Substrate (Thermo Scientific) and enhanced chemiluminescence (G:BOX Chemi XRQ,

511 Syngene).

512

513 ChIP-PCR and ChIP-seq. Chromatin immunoprecipitation was carried out as described by

514 Coughlan et al. (Coughlan et al. 2016) from log phase cultures in $200 \mathrm{ml}$ YPD. Control

515 immunoprecipitations were carried out in the absence of the anti-HA antibody (Mock-IP), and

516 from C. parapsilosis 90-137 without a tagged Cse4 (CTRL). Dilutions of the protein extracts 
517 before immunoprecipitation (Input), and following immunoprecipitation (IP) and mock IP were

518 used to assess binding to CEN1 by PCR amplification, using primers from 5 regions within

519 the predicted CEN1 area, one pair from within the next largest intergenic region on

520 chromosome 1 (chr1:1948277-1955373; to serve as negative control), and a region from

521 within the actin gene ACT1 (Fig. S1, Table S1). ChIP sequencing was performed by Beijing

522 Genomics Institute (BGI) on the BGISEQ500 platform. Approximately 20 million single-end

523 reads (50 bases) were obtained per sample. ChIP-seq reads were mapped to the genome of

524 C. parapsilosis CDC317 (Butler et al. 2009) using the aln/samse algorithm from BWA

525 v0.7.17-r1188 (Li and Durbin 2010), with default parameters. Mapped reads were sorted and

526 indexed with Samtools $v 1.9$ ( $\mathrm{Li}$ et al. 2009) and the read coverage across the genome was

527 computed using Bedtools v2.27.1 (Quinlan and Hall 2010). Genome coverage files were

528 changed into bigwig format using bedGraphToBigWig v4 (Kent et al. 2010) and loaded into

529 Integrated Genomics Viewer (Thorvaldsdóttir et al. 2013) for visualization.

530

531 minION sequencing. One derivative of $C$. parapsilosis 90-137 containing Cse4-HA was

532 sequenced using the minION device from Oxford Nanopore Technologies (ONT). DNA was

533 extracted using the MagJET Genomic DNA Kit \#K2721 from ThermoScientific. Libraries

534 were prepared with the Rapid Sequencing Kit (RSK-SQK004) from ONT and sequenced on

535 a minION flow cell (FLO-MIN106), yielding 30X coverage. Basecalling was performed using

536 Guppy v2.3.7+e041753. Read length and quality was assessed using NanoPlot v1.23.1 (De

537 Coster et al. 2018). NanoFilt v2.3.0 (De Coster et al. 2018) was used to remove reads with a

538 quality score of less than 7 , and the assembly was constructed using canu v1.8 (Koren et al.

539 2017) with options genomeSize=13030174 (to specify the genome size) and -nanopore-raw

540 (for ONT data). Nanopolish v0.11.1 (Loman et al. 2015) was used to improve the consensus

541 accuracy of the assembly and the sequence quality was further improved by incorporating

542 the BGISEQ data from the "input" sample of the ChIP-seq experiment using Pilon v1.23

543 (Walker et al. 2014). The assembly quality was assessed with Quast v4.6.1 (Gurevich et al.

544 2013). Circoletto and Circos v0.69 (Darzentas 2010; Krzywinski et al. 2009) were used to

545 visualise alignments between the $C$. parapsilosis CDC317 reference genome and $C$.

546 parapsilosis 90-137/Cse4-HA assembly. Contigs of less than 1kb and/or contigs mapping to

547 the mitochondrial genome were removed from the assembly.

548

549 Data access

550 All data generated for this project has been deposited in GenBank under the NCBI

551 BioProject ID PRJNA563885. 


\section{ACKNOWLEDGEMENTS}

This work was supported by Science Foundation Ireland (12/IA/1343 (GB) and 13/IA/1910 (KW), https://www.sfi.ie), The Wellcome Trust (102406/Z/13/Z and 109167/Z/15/Z, https://wellcome.ac.uk) and the European Research Council (789341) (KW).

\section{DISCLOSURE DECLARATION}

The authors declare that they have no conflicts of interest.

\section{REFERENCES}

Altschul S. 1990. Basic Local Alignment Search Tool. J Mol Biol 215: 403-410.

Bensasson D, Zarowiecki M, Burt A, Koufopanou V. 2008. Rapid evolution of yeast centromeres in the absence of drive. Genetics 178: 2161-2167.

Burrack LS, Berman J. 2012. Neocentromeres and epigenetically inherited features of centromeres. Chromosome Research 20: 607-619.

Butler G, Rasmussen MD, Lin MF, Santos MAS, Sakthikumar S, Munro CA, Rheinbay E, Grabherr M, Forche A, Reedy JL, et al. 2009. Evolution of pathogenicity and sexual reproduction in eight Candida genomes. Nature 459: 657-662.

Chatterjee G, Sankaranarayanan SR, Guin K, Thattikota Y, Padmanabhan S, Siddharthan $R$, Sanyal K. 2016. Repeat-associated Fission yeast-like regional centromeres in the Ascomycetous budding yeast Candida tropicalis. PLOS Genet 12: e1005839.

Coughlan AY, Hanson SJ, Byrne KP, Wolfe KH. 2016. Centromeres of the yeast Komagataella phaffii (Pichia pastoris) have a simple Inverted-Repeat structure. Genome Biol Evol 8: 2482-2492.

Darzentas N. 2010. Circoletto: visualizing sequence similarity with Circos. Bioinformatics 26: 2620-2621.

De Coster W, D'Hert S, Schultz DT, Cruts M, Van Broeckhoven C. 2018. NanoPack: visualizing and processing long-read sequencing data. Bioinformatics 34: 2666-2669.

Donovan PD, Schröder MS, Higgins DG, Butler G. 2016. Identification of Non-Coding RNAs in the Candida parapsilosis species group. PLoS One 11: e0163235.

Douglass AP, Offei B, Braun-Galleani S, Coughlan AY, Martos AAR, Ortiz-Merino RA, Byrne $\mathrm{KP}$, Wolfe KH. 2018. Population genomics shows no distinction between pathogenic Candida krusei and environmental Pichia kudriavzevii: One species, four names. PLoS Pathog 14: e1007138.

Drillon G, Carbone A, Fischer G. 2014. SynChro: a fast and easy tool to reconstruct and visualize synteny blocks along eukaryotic chromosomes. PLoS One 9: e92621. 
Dujon B, Sherman D, Fischer G, Durrens P, Casaregola S, Lafontaine I, De Montigny J, Marck C, Neuvéglise C, Talla E, et al. 2004. Genome evolution in yeasts. Nature 430: 35-44.

Fitzpatrick DA, Logue ME, Stajich JE, Butler G. 2006. A fungal phylogeny based on 42 complete genomes derived from supertree and combined gene analysis. BMC Evol Biol 6: 99.

Fitzpatrick DA, O'Gaora P, Byrne KP, Butler G. 2010. Analysis of gene evolution and metabolic pathways using the Candida Gene Order Browser. BMC Genomics 11: 290.

Folco HD, Pidoux AL, Urano T, Allshire RC. 2008. Heterochromatin and RNAi are required to establish CENP-A chromatin at centromeres. Science 319: 94-97.

Fournier P, Abbas A, Chasles M, Kudla B, Ogrydziak DM, Yaver D, Xuan JW, Peito A, Ribet AM, Feynerol C. 1993. Colocalization of centromeric and replicative functions on autonomously replicating sequences isolated from the yeast Yarrowia lipolytica. Proc Natl Acad Sci U S A 90: 4912-4916.

Friedman S, Freitag M. 2017. Centrochromatin of Fungi. Prog Mol Subcell Biol 56: 85-109.

Furuyama S, Biggins S. 2007. Centromere identity is specified by a single centromeric nucleosome in budding yeast. Proc Natl Acad Sci U S A 104: 14706-14711.

Gordon JL, Byrne KP, Wolfe KH. 2011. Mechanisms of chromosome number evolution in yeast. PLoS Genet 7: e1002190.

Gurevich A, Saveliev V, Vyahhi N, Tesler G. 2013. QUAST: quality assessment tool for genome assemblies. Bioinformatics 29: 1072-1075.

Henikoff S, Ahmad K, Malik HS. 2001. The centromere paradox: stable inheritance with rapidly evolving DNA. Science 293: 1098-1102.

Henikoff S, Henikoff JG. 2012. "Point" centromeres of Saccharomyces harbor single centromere-specific nucleosomes. Genetics 190: 1575-1577.

Janbon G, Ormerod KL, Paulet D, Byrnes EJ 3rd, Yadav V, Chatterjee G, Mullapudi N, Hon C-C, Billmyre RB, Brunel F, et al. 2014. Analysis of the genome and transcriptome of Cryptococcus neoformans var. grubii reveals complex RNA expression and microevolution leading to virulence attenuation. PLOS Genet 10: e1004261.

Kapoor S, Zhu L, Froyd C, Liu T, Rusche LN. 2015. Regional centromeres in the yeast Candida lusitaniae lack pericentromeric heterochromatin. Proc Natl Acad Sci U S A 112: 12139-12144.

Kasinathan S, Henikoff S. 2018. Non-B-Form DNA Is Enriched at Centromeres. Mol Biol Evol 35: 949-962.

Kent WJ, Zweig AS, Barber G, Hinrichs AS, Karolchik D. 2010. BigWig and BigBed: enabling browsing of large distributed datasets. Bioinformatics 26: 2204-2207.

Ketel C, Wang HSW, McClellan M, Bouchonville K, Selmecki A, Lahav T, Gerami-Nejad M, Berman J. 2009. Neocentromeres form efficiently at multiple possible loci in Candida albicans. PLoS Genet 5: e1000400.

Kitada K, Yamaguchi E, Hamada K, Arisawa M. 1997. Structural analysis of a Candida glabrata centromere and its functional homology to the Saccharomyces cerevisiae 
centromere. Current Genetics 31: 122-127.

Kobayashi N, Suzuki Y, Schoenfeld LW, Müller CA, Nieduszynski C, Wolfe KH, Tanaka TU. 2015. Discovery of an unconventional centromere in budding yeast redefines evolution of point centromeres. Curr Biol 25: 2026-2033.

Koren A, Tsai H-J, Tirosh I, Burrack LS, Barkai N, Berman J. 2010. Epigenetically-inherited centromere and neocentromere DNA replicates earliest in S-phase. PLoS Genet 6: e1001068.

Koren S, Walenz BP, Berlin K, Miller JR, Bergman NH, Phillippy AM. 2017. Canu: scalable and accurate long-read assembly via adaptive k-mer weighting and repeat separation. Genome Res 27: 722-736.

Krzywinski M, Schein J, Birol I, Connors J, Gascoyne R, Horsman D, Jones SJ, Marra MA. 2009. Circos: an information aesthetic for comparative genomics. Genome Res 19: $1639-1645$.

Lefrançois P, Auerbach RK, Yellman CM, Roeder GS, Snyder M. 2013. Centromere-like regions in the budding yeast genome. PLoS Genet 9: e1003209.

Li H, Durbin R. 2010. Fast and accurate long-read alignment with Burrows-Wheeler transform. Bioinformatics 26: 589-595.

Li H, Handsaker B, Wysoker A, Fennell T, Ruan J, Homer N, Marth G, Abecasis G, Durbin R, 1000 Genome Project Data Processing Subgroup. 2009. The Sequence Alignment/Map format and SAMtools. Bioinformatics 25: 2078-2079.

Locke DP, Hillier LW, Warren WC, Worley KC, Nazareth LV, Muzny DM, Yang S-P, Wang Z, Chinwalla AT, Minx P, et al. 2011. Comparative and demographic analysis of orangutan genomes. Nature 469: 529-533.

Loman NJ, Quick J, Simpson JT. 2015. A complete bacterial genome assembled de novo using only nanopore sequencing data. Nat Methods 12: 733-735.

Lombardi L, Oliveira-Pacheco J, Butler G. 2019a. Plasmid-based CRISPR-Cas9 gene editing in multiple Candida species. mSphere 4: e00125-19.

Lombardi L, Turner SA, Zhao F, Butler G. 2017. Gene editing in clinical isolates of Candida parapsilosis using CRISPR/Cas9. Sci Rep 7: 8051.

Lombardi L, Zoppo M, Rizzato C, Bottai D, Hernandez AG, Hoyer LL, Tavanti A. 2019b. Characterization of the Candida orthopsilosis agglutinin-like sequence ( $A L S)$ genes. PLoS One 14: e0215912.

Lorenz R, Bernhart SH, Höner Zu Siederdissen C, Tafer H, Flamm C, Stadler PF, Hofacker IL. 2011. ViennaRNA Package 2.0. Algorithms Mol Biol 6: 26.

Lynch DB, Logue ME, Butler G, Wolfe KH. 2010. Chromosomal G + C content evolution in yeasts: systematic interspecies differences, and GC-poor troughs at centromeres. Genome Biol Evol 2: 572-583.

Maguire SL, ÓhÉigeartaigh SS, Byrne KP, Schröder MS, O'Gaora P, Wolfe KH, Butler G. 2013. Comparative genome analysis and gene finding in Candida species using CGOB. Mol Biol Evol 30: 1281-1291.

Malik HS, Henikoff S. 2009. Major evolutionary transitions in centromere complexity. Cell 
138: $1067-1082$.

Mattei S, Sampaolese B, De Santis P, Savino M. 2002. Nucleosome organization on Kluyveromyces lactis centromeric DNAs. Biophys Chem 97: 173-187.

Meluh PB, Yang P, Glowczewski L, Koshland D, Mitchell Smith M. 1998. Cse4p Is a component of the core centromere of Saccharomyces cerevisiae. Cell 94: 607-613.

Morales L, Noel B, Porcel B, Marcet-Houben M, Hullo M-F, Sacerdot C, Tekaia F, Leh-Louis V, Despons L, Khanna V, et al. 2013. Complete DNA sequence of Kuraishia capsulata illustrates novel genomic features among budding yeasts (Saccharomycotina). Genome Biol Evol 5: 2524-2539.

Nesmelova IV, Hackett PB. 2010. DDE transposases: Structural similarity and diversity. Adv Drug Deliv Rev 62: 1187-1195.

Ohama T, Suzuki T, Mori M, Osawa S, Ueda T, Watanabe K, Nakase T. 1993. Nonuniversal decoding of the leucine codon CUG in several Candida species. Nucleic Acids Research 21: 4039-4045.

Ohkuni K, Kitagawa K. 2011. Endogenous transcription at the centromere facilitates centromere activity in budding yeast. Curr Biol 21: 1695-1703.

Padmanabhan S, Thakur J, Siddharthan R, Sanyal K. 2008. Rapid evolution of Cse4p-rich centromeric DNA sequences in closely related pathogenic yeasts, Candida albicans and Candida dubliniensis. Proc Natl Acad Sci U S A 105: 19797-19802.

Pearson CE, Zorbas H, Price GB, Zannis $\square$ Hadjopoulos M. 1996. Inverted repeats, stem $\square$ loops, and cruciforms: Significance for initiation of DNA replication. Journal of Cellular Biochemistry 63: 1-22.

Pryszcz LP, Németh T, Saus E, Ksiezopolska E, Hegedüsová E, Nosek J, Wolfe KH, Gacser A, Gabaldón T. 2015. The genomic aftermath of hybridization in the opportunistic pathogen Candida metapsilosis. PLoS Genet 11: e1005626.

Purgato S, Belloni E, Piras FM, Zoli M, Badiale C, Cerutti F, Mazzagatti A, Perini G, Della Valle G, Nergadze SG, et al. 2015. Centromere sliding on a mammalian chromosome. Chromosoma 124: 277-287.

Quinlan AR, Hall IM. 2010. BEDTools: a flexible suite of utilities for comparing genomic features. Bioinformatics 26: 841-842.

Riccombeni A, Vidanes G, Proux-Wéra E, Wolfe KH, Butler G. 2012. Sequence and analysis of the genome of the pathogenic yeast Candida orthopsilosis. PLoS One 7: e35750.

Rocchi M, Archidiacono N, Schempp W, Capozzi O, Stanyon R. 2012. Centromere repositioning in mammals. Heredity 108: 59-67.

Roy B, Sanyal K. 2011. Diversity in requirement of genetic and epigenetic factors for centromere function in fungi. Eukaryot Cell 10: 1384-1395.

Sanyal K, Baum M, Carbon J. 2004. Centromeric DNA sequences in the pathogenic yeast Candida albicans are all different and unique. Proc Nat Acad Sci (USA) 101: 1137411379.

Schröder MS, Martinez de San Vicente K, Prandini THR, Hammel S, Higgins DG, Bagagli E, Wolfe KH, Butler G. 2016. Multiple origins of the pathogenic yeast Candida orthopsilosis 
by separate hybridizations between two parental species. PLoS Genet 12: e1006404.

Schubert I. 2018. What is behind "centromere repositioning"? Chromosoma 127: 229-234.

Schulman I, Bloom KS. 1991. Centromeres: an integrated protein/DNA complex required for chromosome movement. Annu Rev Cell Biol 7: 311-336.

Shen X-X, Opulente DA, Kominek J, Zhou X, Steenwyk JL, Buh KV, Haase MAB, Wisecaver $\mathrm{JH}$, Wang M, Doering DT, et al. 2018. Tempo and mode of genome evolution in the budding yeast subphylum. Cell 175: 1533-1545.

Sreekumar L, Jaitly P, Chen Y, Thimmappa BC, Sanyal A, Sanyal K. 2019. Cis and trans chromosomal interactions define pericentric boundaries in the absence of conventional heterochromatin. Genetics 212: 1121-1132.

Stanyon R, Rocchi M, Capozzi O, Roberto R, Misceo D, Ventura M, Cardone MF, Bigoni F, Archidiacono N. 2008. Primate chromosome evolution: ancestral karyotypes, marker order and neocentromeres. Chromosome Res 16: 17-39.

Sun S, Yadav V, Billmyre RB, Cuomo CA, Nowrousian M, Wang L, Souciet J-L, Boekhout T, Porcel B, Wincker $P$, et al. 2017. Fungal genome and mating system transitions facilitated by chromosomal translocations involving intercentromeric recombination. PLoS Biol 15: e2002527.

Tavanti A, Davidson AD, Gow NAR, Maiden MCJ, Odds FC. 2005. Candida orthopsilosis and Candida metapsilosis spp. nov. to replace Candida parapsilosis groups II and III. $J$ Clin Microbiol 43: 284-292.

Thakur J, Sanyal K. 2013. Efficient neocentromere formation is suppressed by gene conversion to maintain centromere function at native physical chromosomal loci in Candida albicans. Genome Res 23: 638-652.

Thorvaldsdóttir H, Robinson JT, Mesirov JP. 2013. Integrative Genomics Viewer (IGV): highperformance genomics data visualization and exploration. Brief Bioinform 14: 178-192.

Turner SA, Ma Q, Ola M, Martinez de San Vicente K, Butler G. 2018. Dal81 regulates expression of arginine metabolism genes in Candida parapsilosis. mSphere 3. http://dx.doi.org/10.1128/mSphere.00028-18.

Vakirlis N, Sarilar V, Drillon G, Fleiss A, Agier N, Meyniel J-P, Blanpain L, Carbone A, Devillers H, Dubois K, et al. 2016. Reconstruction of ancestral chromosome architecture and gene repertoire reveals principles of genome evolution in a model yeast genus. Genome Res 26: 918-932.

Wade CM, Giulotto E, Sigurdsson S, Zoli M, Gnerre S, Imsland F, Lear TL, Adelson DL, Bailey E, Bellone RR, et al. 2009. Genome sequence, comparative analysis, and population genetics of the domestic horse. Science 326: 865-867.

Walker BJ, Abeel T, Shea T, Priest M, Abouelliel A, Sakthikumar S, Cuomo CA, Zeng Q, Wortman J, Young SK, et al. 2014. Pilon: an integrated tool for comprehensive microbial variant detection and genome assembly improvement. PLoS One 9: e112963.

Yamane T, Ogawa T, Matsuoka M. 2008. Derivation of consensus sequence for protein binding site in Yarrowia lipolytica centromere. J Biosci Bioeng 105: 671-674. 


\section{Figure legends}

Fig.1 C. parapsilosis centromeres consist of unique mid regions surrounded by partially conserved Inverted Repeats.

A. Dot matrix plot comparing the putative centromere sequences in C. parapsilosis. Centromere regions (Supplemental_Table_S2) were concatenated and are delineated by dark blue lines. Inverted repeats (Right, IRR and left, IRL) are separated with cyan lines. Each dot represents a 25-bp window. Inverted sequences are shown in red, and direct repeats in black.

B. Diagrammatic representation of the information in (A). Regions that are conserved among chromosomes are shown in black. Locations of inverted repeats (> 75\% DNA sequence identity) are shown with white arrows. The mid regions are illustrated in different colors that indicate that each of them has a unique sequence. Adjacent genes are shown in gray. Each region shown is approximately $10 \mathrm{~kb}$ in length.

C. Three copies of an HA tag were introduced into both alleles of the endogenous CSE4 gene in C. parapsilosis CLIB214 and 90-137 using CRISPR-Cas9 editing. The gene was cut between Glycine 69 and Glycine 70, and a repair template containing the HA tags was inserted by homologous recombination. The construct was confirmed by sequencing.

D. Visualization of the ChIP-seq signal across all chromosomes (Chr) in Cse4-tagged derivatives of $C$. parapsilosis CLIB214 and 90-137. In = Input (before immunoprecipitation), IP1 and IP2 show two independent immunoprecipitation replicates from each strain. Strains derived from C. parapsilosis CLIB214 are shown in blue, and from 90-137 in purple. There is one signal per chromosome in the IP samples, identifying the centromere, except for chromosome 7, for which the rDNA locus (black asterisk) also generates a signal. The Xaxis in each plot is the chromosome coordinates and the $\mathrm{Y}$-axis is the number of reads mapping to a position. The maximum scale for $C$. parapsilosis CLIB214 is restricted to reduce the signal from the rDNA. Data is visualized using Integrated Genomics Viewer (IGV) (Thorvaldsdóttir et al. 2013).

Fig. 2 Natural polymorphisms for centromere location in C. parapsilosis 
The ChIP-Seq data from Fig. 1D is shown in more detail, and the neocentromeres are highlighted with black boxes. The order of the tracks is the same in each panel, but is labelled for CEN1 only. The top track shows the location of $C$. parapsilosis protein coding genes. The second track shows the IR sequences only (red), with an arrow indicating the direction of the repeat. The extent of the regions conserved between chromosomes is not shown. ChIP-Seq read coverage is plotted in blue for $C$. parapsilosis CLIB214, and purple for $C$. parapsilosis 90-137. Two independent immunoprecipitation experiments were carried out per strain (IP1 and IP2). Only one control is shown; the total chromatin from $C$. parapsilosis CLIB214 (Input). The equivalent data for C. parapsilosis 90-137, and for an experiment with no tagged Cse4, is available at GEO, accession number GSE136854. The bottom track (gray) shows gene expression measured by RNA-seq during growth in YPD (taken from SRR6458364 from (Turner et al. 2018)). The read depth scale is indicated in brackets; the total number of reads varied in each experiment. The maximum scale for $C$. parapsilosis CLIB214 is restricted to 500 to reduce the signal from the rDNA. The RNA expression data is plotted on a log scale. The apparent dips in coverage at the centromeres in the input data is likely to be an artefact of the mapping procedure because reads that map to more than one site in the genome were discarded. Some reads are also incorrectly mapped to non-identical repeat sequences, resulting in a small Cse4 signal at CEN5 in 90137. All data is visualized using IGV.

Fig. 3 Lack of rearrangements at CEN1 and CEN5 in C. parapsilosis 90-137/Cse4-HA.

The Circos plot compares the 8 chromosomes of the reference strain C. parapsilosis CDC317 (gray, left) to the 12 largest minION scaffolds from C. parapsilosis 90-137 (white, right). Centromeres are marked by black bands. Most chromosomes are collinear, including chromosome 1 (assembled in two contigs in 90-137, contig 1 and contig 16) and chromosome 5 (contig 2). There is an apparent translocation between chromosomes 3 and 4 (contig 3 and contig 8 ) at a repetitive gene that is near (but not at) the centromere. This may represent an error in the reference assembly, or a natural structural polymorphism.

Fig. 4. Identification of centromeres and centromere-proximal rearrangements in $C$. orthopsilosis and C. metapsilosis

A. Cartoon of centromere structure in C. orthopsilosis 90-125 (Lombardi et al. 2019b). All mid regions are unique and are shown in different colors. Sequences in black are conserved among chromosomes. IRs are shown with white arrows, and adjacent genes are shown with 
gray boxes. Putative transposases with DDE domains are indicated. More detail is provided in Supplemental_Fig_S2 and Supplemental_Table_S2.

B and C. Synteny relationship between C. parapsilosis and C. orthopsilosis. SynChro (Drillon et al. 2014) was used (delta value of 2) to identify potential orthologs (reciprocal best hits, $\mathrm{RBHs})$, represented by colored lines in the two species, and to generate synteny maps. $\mathrm{B}$. Location of RBHs on C. orthopsilosis chromosomes. The approximate location of the putative centromeres are indicated with a gray polygon. C. C. parapsilosis chromosomes, colored with respect to the $\mathrm{RBH}$ from $C$. orthopsilosis. The location of the C. parapsilosis centromeres are indicated with an offset white circle. The location of syntenic $C$. orthopsilosis centromeres is shown in more detail in Fig. 5.

D. Cartoon of centromere structure in C. metapsilosis. Sequences in black are conserved among chromosomes. IRs are shown with white arrows, which are sometimes fragmented and overlapping. Mid-core regions from some CENs are similar in sequence $(>60 \%)$ and are shown in the same color. Adjacent genes are shown with gray boxes. More detail is provided in Supplemental_Fig_S2 and Supplemental_Table_S2.

E and F. Synteny relationship between C. parapsilosis and C. metapsilosis. E. Location of $\mathrm{RBH}$ s on $C$. metasilosis chromosomes. The approximate location of the putative $C$. metapsilosis centromeres are indicated with a gray star (centromeres were not identified on scaffolds 4 and 8). F. C. parapsilosis chromosomes, colored with respect to the RBH from $C$. metapsilosis. The location of the $C$. parapsilosis centromeres are indicated with a white circle. The approximate location of syntenic $C$. metapsilosis centromeres are shown by name and with gray stars. The same colors are used for $C$. orthopsilosis $(\mathrm{B})$ and $C$. metapsilosis (D). This does not indicate that synteny is completely conserved between these species; it is a feature of SynChro, which carries out pairwise comparisons.

Fig. 5 Interspecies synteny breakpoints occur at centromeres

Synteny between $C$. parapsilosis and $C$. orthopsilosis was visualized using SynChro (Drillon et al. 2014), with a delta value of 2. Changing delta values had minor effects on predicted synteny. A diagrammatic representation of each $C$. parapsilosis chromosome, colored as in Fig. $4 \mathrm{C}$, is shown to scale at the top of each panel. The lower sections of each panel show the gene order around the centromere. 
A-F. Gene order around the 8 centromeres in C. parapsilosis compared to C. orthopsilosis. The bottom row in each panel shows gene order on the C. parapsilosis chromosome, and the $8 \mathrm{C}$. orthopsilosis chromosomes are shown above. Each gene is indicated by a colored dot, and RBH are joined by lines. Syntenic blocks are surrounded with a box. Centromeres are shown by large black circles. The chromosome number is indicated at the side of each panel. The names of some genes are shown for orientation purposes. The prefix "CORTO" has been removed from $C$. orthopsilosis genes and "CPAR2_" from C. parapsilosis genes for brevity. The color of the dots indicates the similarity of the proteins. Non inverted RBH are shown in green, ranging from darkest ( $>90 \%$ similarity) to lightest ( $<30 \%$ similarity), and inverted orthologs are shown in red. Genes without RBH orthologs are shown in blue. Genes in gray were not identified as RBH by SynChro, but were identified using CGOB (Maguire et al. 2013; Fitzpatrick et al. 2010).

Fig. 6. Organization of centromeres in Saccharomycotina species.

The phylogeny is adapted from Shen et al (Shen et al. 2018). The size indicated on the centromeres refers to the region bound by Cse4 where known, or else where predicted bioinformatically, except for the Saccharomycetaceae where the size of the point centromere is shown. Solid color indicates conservation of sequence across centromeres in the same species, whereas a color gradient indicates unique sequences. Inverted repeats (IR) are shown with arrows, and Ty clusters as red and green boxes. Black circles show known (solid) or predicted (open) early firing origins of replication (see text for details). Point centromeres are conserved across the Saccharomycetaceae except for the Naumovozyma lineage, which have different sequences. Question marks indicate that localization of Cse4 nucleosomes has not been determined.

Fig. S1 Expression of Cse4-HA and ChIP-PCR

A. Expression of Cse4-HA in C. parapsilosis 90-137 was detected by Western blot using anti-HA antibody. Lane M: PageRuler Plus Prestained Protein ladder (Thermo Scientifics). Sizes are shown in kD. Lanes 1 and 2: protein extract from two independent Cse4-HA tagged derivatives of $C$. parapsilosis 90-137 Lane 3: protein extract from untagged $C$. parapsilosis 90-137. The protein size is expected to be $49.4 \mathrm{kD}$.

B. Tagging Cse4 does not interfere with growth. The tag was introduced at a position that was predicted to be unlikely to interfere with the function of Cse4. Two Cse4-tagged derivatives of $C$. parapsilosis 90-137 (N1 and N2) grow as well as the parental strain $C$. 
parapsilosis 90-137 on YPD. Serial dilutions of cells were spotted on YPD agar and grown for $48 \mathrm{~h}$ at $30^{\circ} \mathrm{C}$.

C. ChIP-PCR from C. parapsilosis CEN1. N1 and N2 are Cse4-tagged derivatives, and CTRL is the untagged strain. PCR amplification was carried out using 5 pairs of primers from within the core region of CEN1 shown in red (Table S1), one pair from an adjacent intergenic region on Chromosome 1 (OUT) and one pair from ACT1. IP = HA immunoprecipitation with anti-HA; INPUT = protein extract before immunoprecipitation; mock IP = no anti-HA antibody used. The target PCR products in the core region are marked with a white asterisk. Some smaller non-specific products were also obtained.

All primer pairs from within CEN1 amplified the expected size fragments from total chromatin (Input) from untagged C. parapsilosis 90-137 and from two Cse4-tagged strains. Some nonspecific PCR products were also amplified. The CEN1-specific primers also amplified sequences from anti-HA chromatin immunoprecipitates in the tagged strains (N1 and N2), but not in the control untagged strain (CTRL). The OUT and ACT1 primers amplified products from the input samples only. Cse4 therefore localizes to the proposed CEN1. D. Sequence of repair template used to introduce HA tags.

Fig. S2. Dot matrix plots showing sequence conservation around $C$. orthopsilosis (A) and $C$. metapsilosis centromeres (B). Centromeres are delineated by dark blue lines. Inverted repeats (Right, IRR and left, IRL) are separated with cyan lines. Each dot represents identity of 25-bp. Inverted sequences are shown in red, and direct repeats in black.

Fig. S3 Rearrangements at putative centromeres in L. elongisporus

Synteny relationship between C. parapsilosis and L. elongisporus identified using SynChro. A. Location of hits on L. elongisporus chromosomes. The approximate location of the putative $L$. elongisporus centromeres are indicated with white stars. Three proposed candidates (marked with white X's) are unlikely to represent centromeres because they are either adjacent to the rDNA (scaffold 9), or on regions that are strongly transcribed (scaffolds 7 and 10). The other proposed centromeres are unique, when only the untranscribed regions are included (Donovan et al. 2016).

B. C. parapsilosis chromosomes, colored with respect to the RBHs from L. elongisporus. The location of the putative C. parapsilosis centromeres are indicated with a white circle. The location of syntenic $L$. elongisporus centromeres (where identified) are indicated by name and with a white star.

Fig S4. Ancestral reconstruction. 
A. The genome structure of the ancestor of C. parapsilosis and C. orthopsilosis (Ancestor 1, A1) was inferred using AnChro (Vakirlis et al. 2016). The syntenic relationship between A1 and C. parapsilosis or C. orthopsilosis (determined using SynChro) is shown (see Fig. 4). $B$. The genome structure of the ancestor of A1 and C. metapsilosis (Ancestor 2, A2) was inferred as in (A).

C. Interchromosomal breaks were identified by pairwise comparisons of synteny maps from SynChro. Thirteen breaks were identified between C. parapsilosis and C. orthopsilosis. By comparing with $\mathrm{A} 1,7$ were placed on the $\mathrm{C}$. orthopsilosis branch, and 4 on the $\mathrm{C}$. parapsilosis branch. Two (shown in parentheses) could not be placed. Similar comparisons are shown with Ancestor A2. The phylogenetic relationship between the species is taken from Pryszcz et al (2015).

Table S1. List of primers used.

Table S2. Coordinates of centromeres in C. parapsilosis, C. orthopsilosis and $C$. metapsilosis. Numbers for $C$. parapsilosis (CDC317) and C. metapsilosis (hybrid) refer to the current assembly for each, available at CGOB (Fitzpatrick et al. 2010). The C. orthopsilosis locations refer to the minION assembly of $C$. orthopsilosis 90-125 from Lombardi et al (Lombardi et al. 2019b). 
A

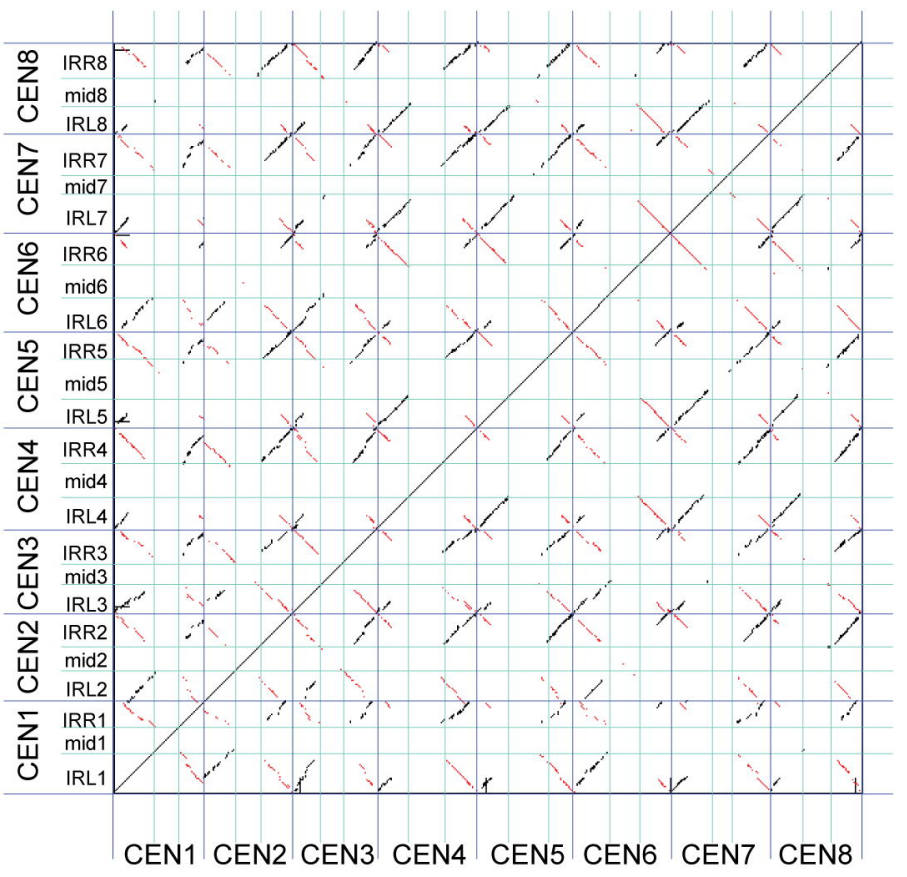

C

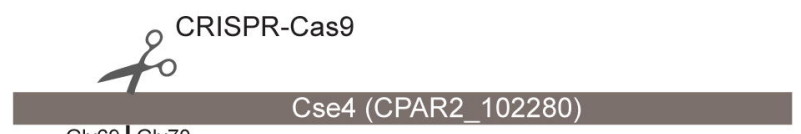

Gly69|Gly70

\section{HA - HA $-H A$}

\section{B}

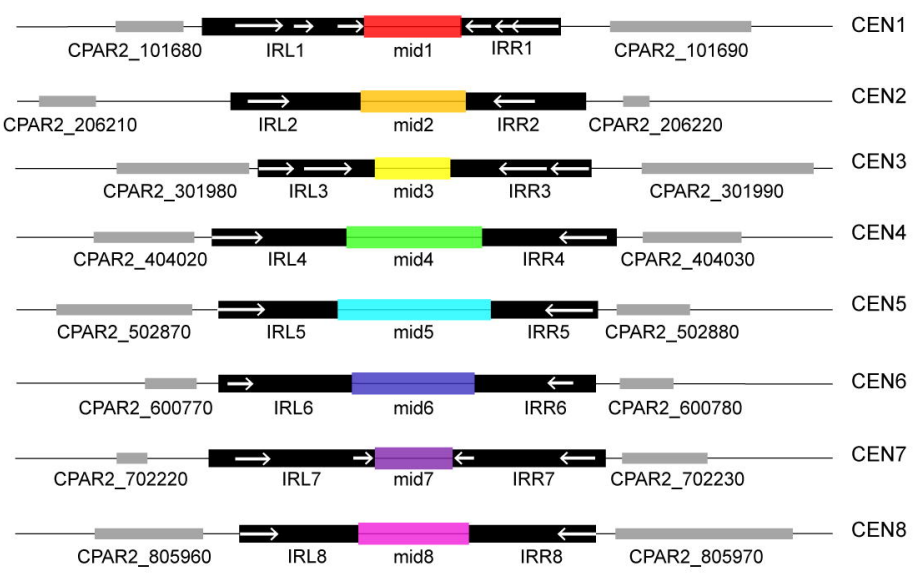

D

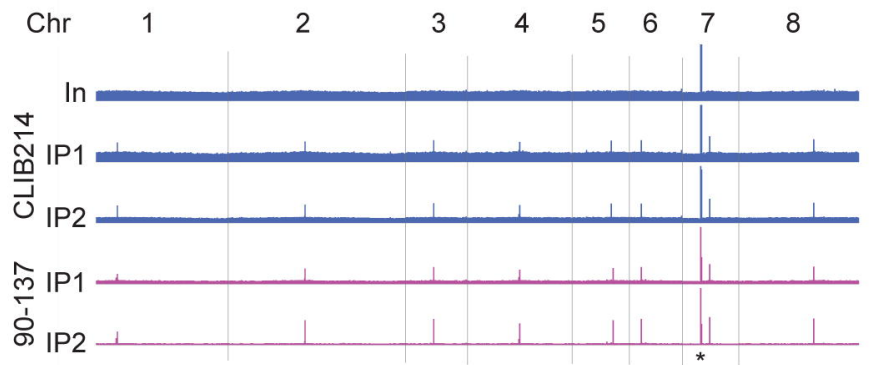



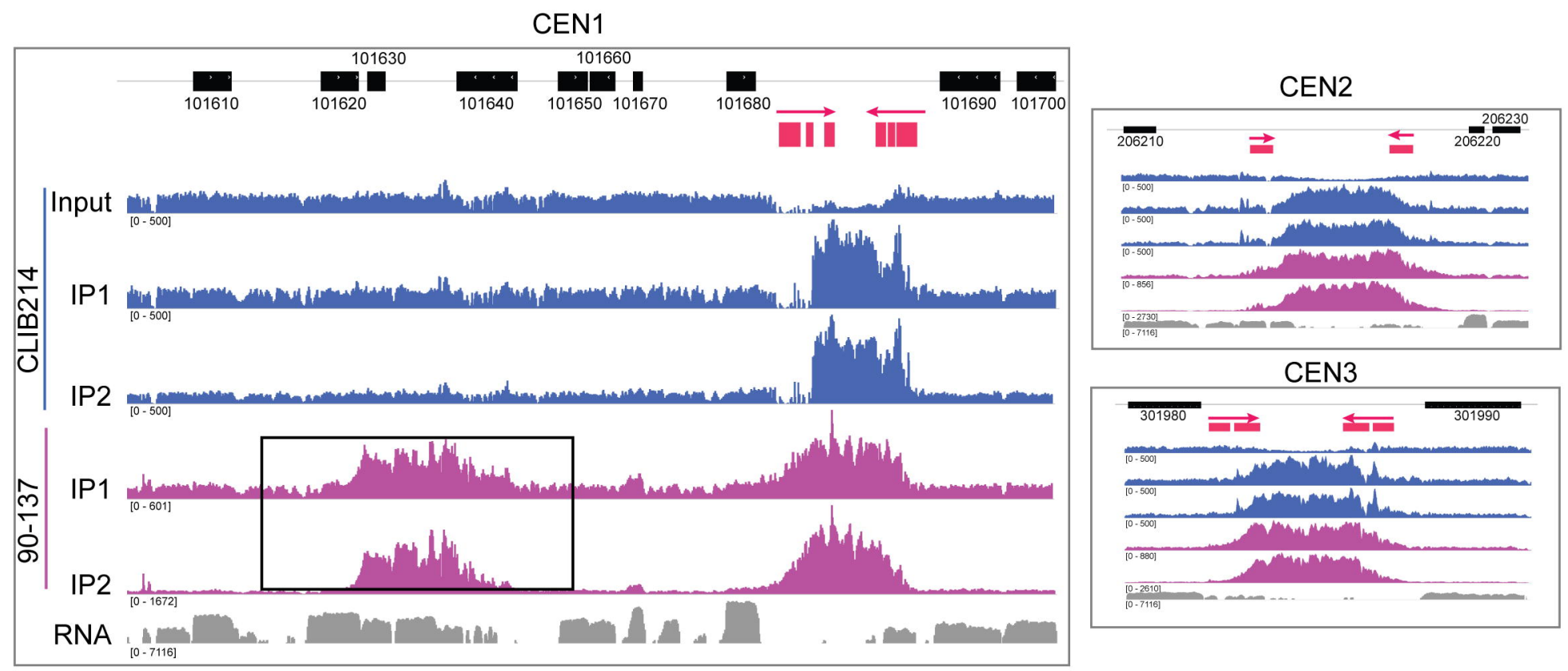

\section{CEN5}
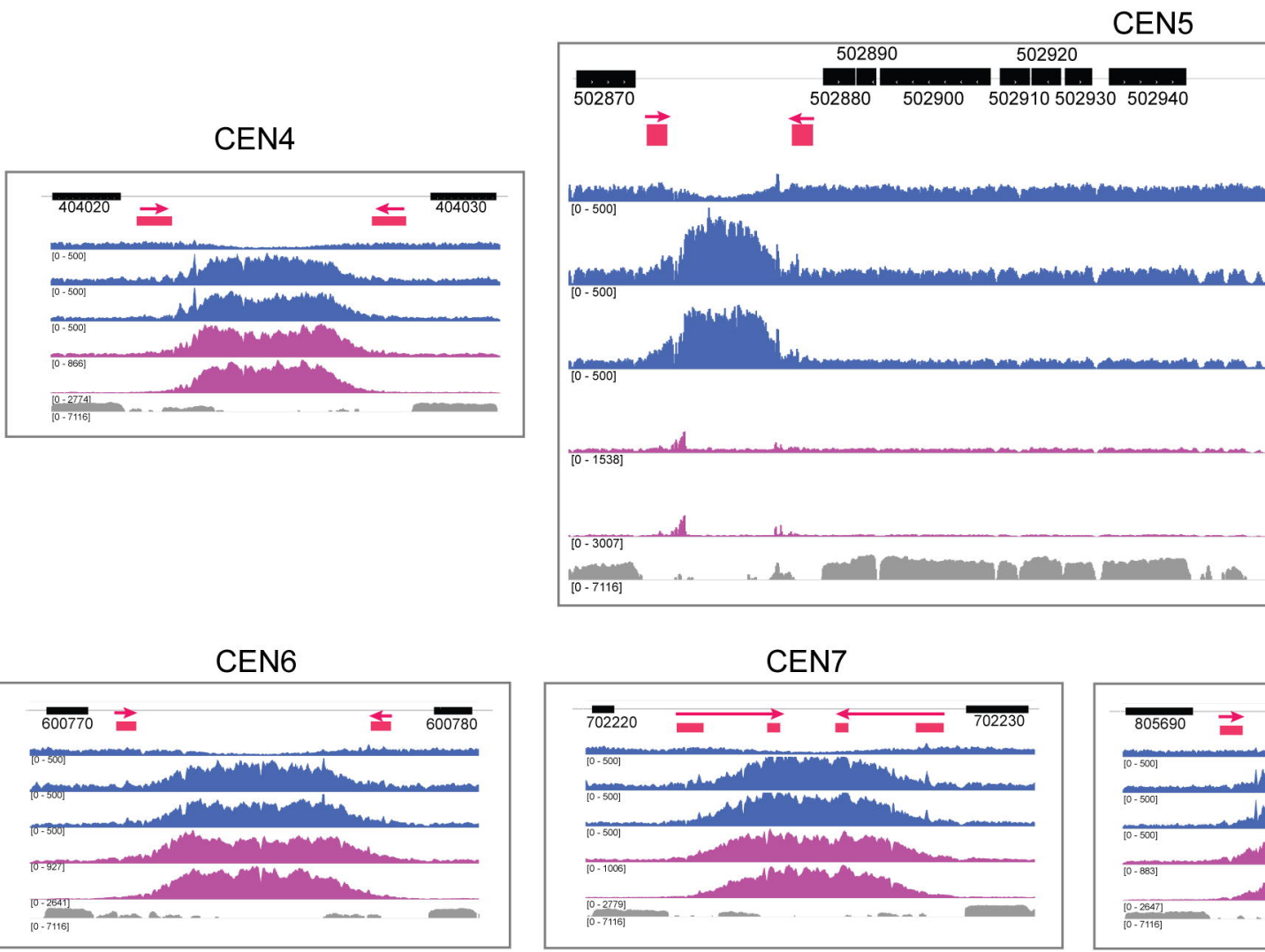

10502930502940

\section{0}

$502960502970 \quad 502980$

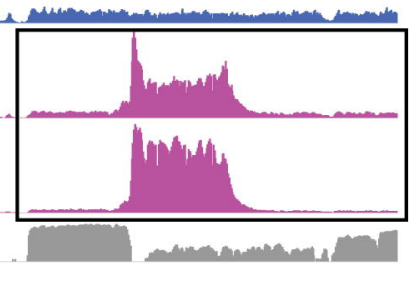

CEN6

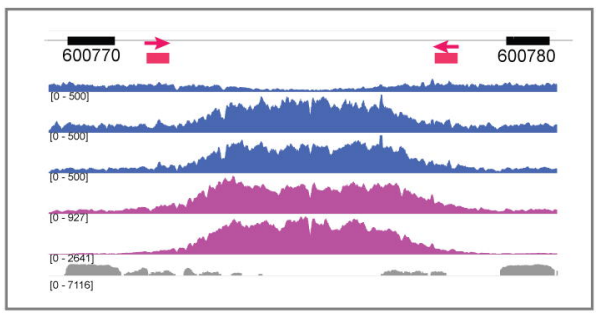




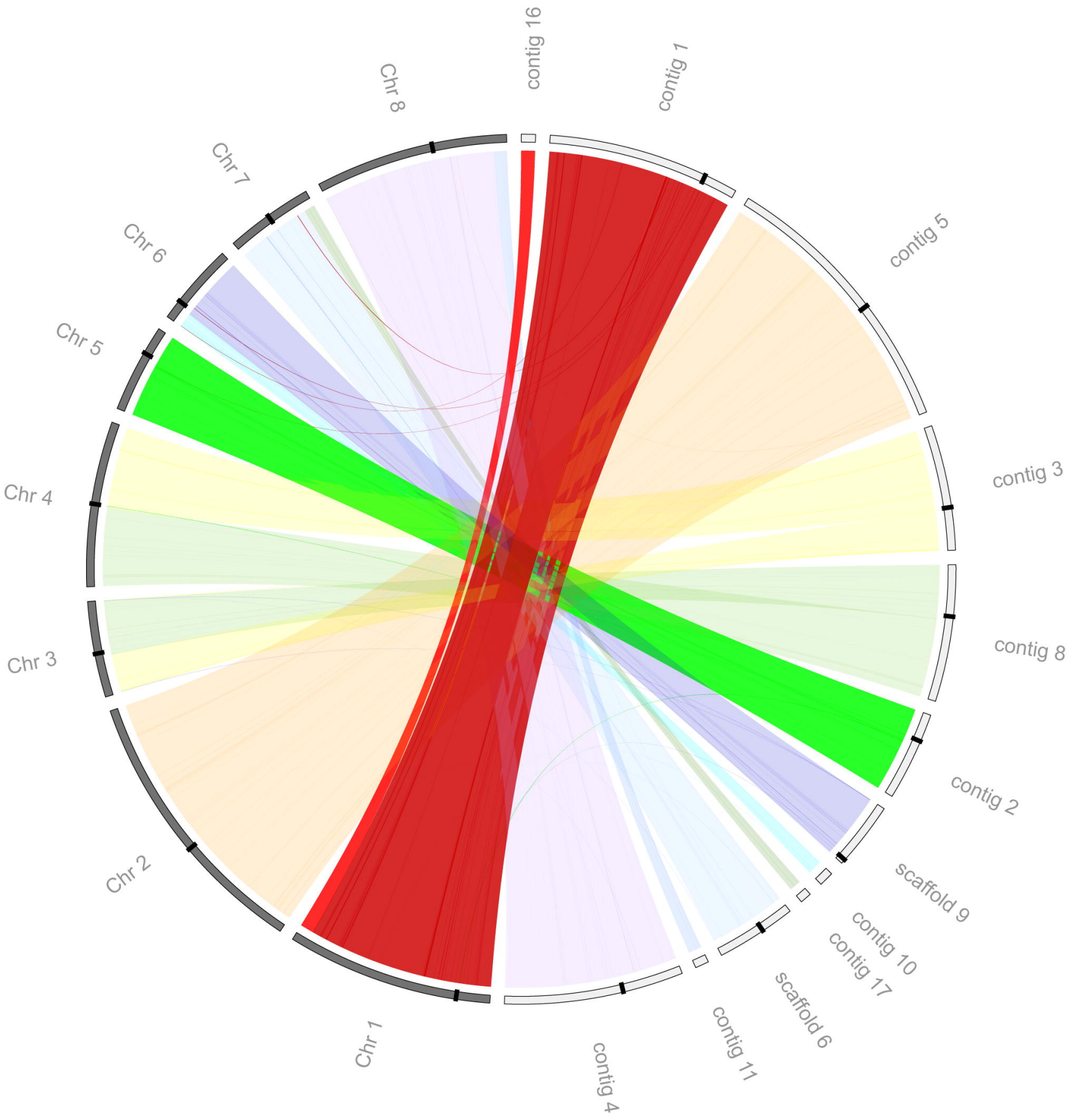


A C. orthopsilosis centromere structure

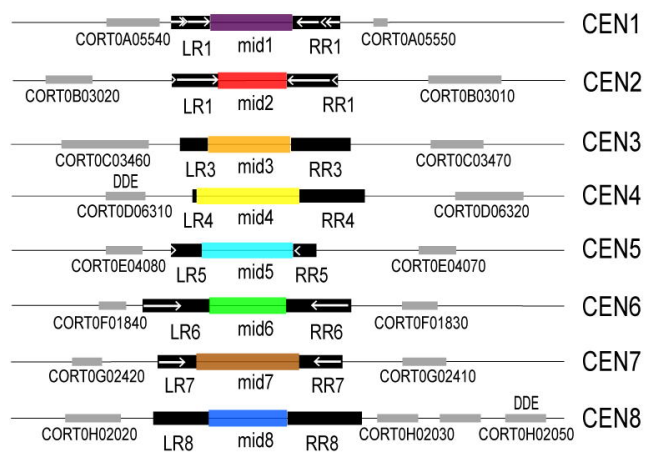

B C. orthopsilosis

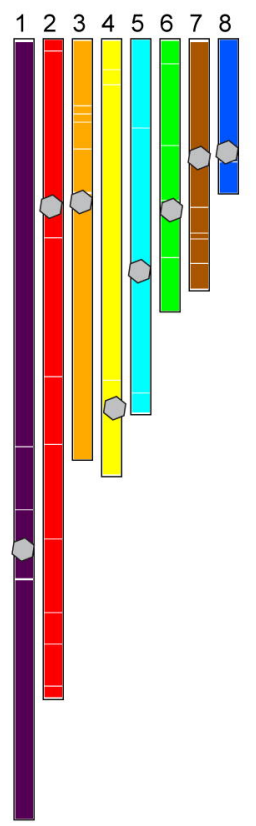

C C. parapsilosis synteny

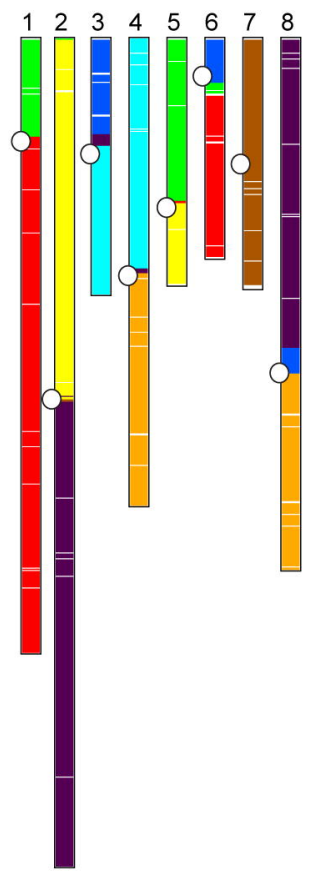

D C. metapsilosis centromere structure

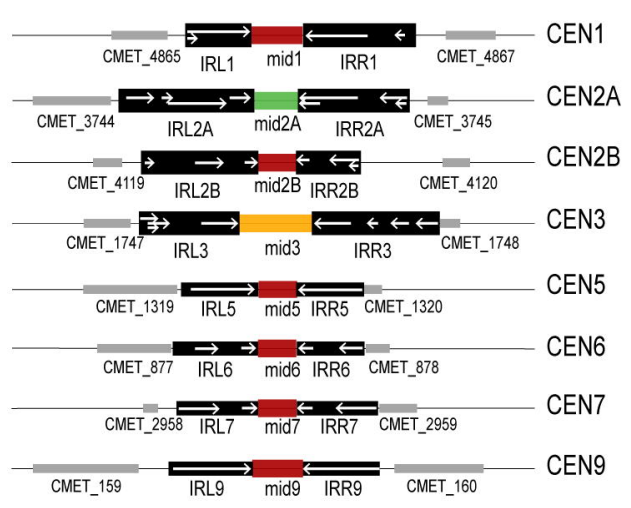

\section{E C. metapsilosis \\ F C. parapsilosis synteny}

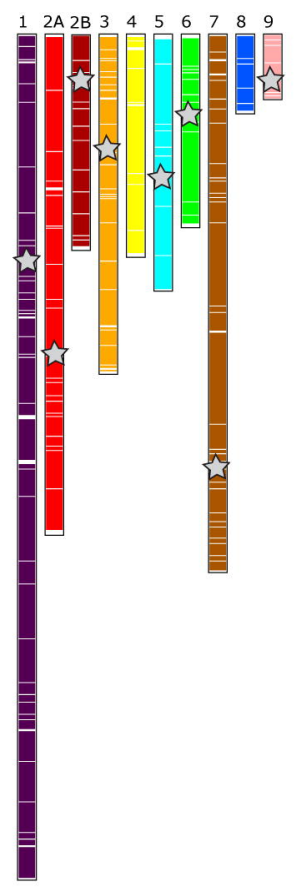

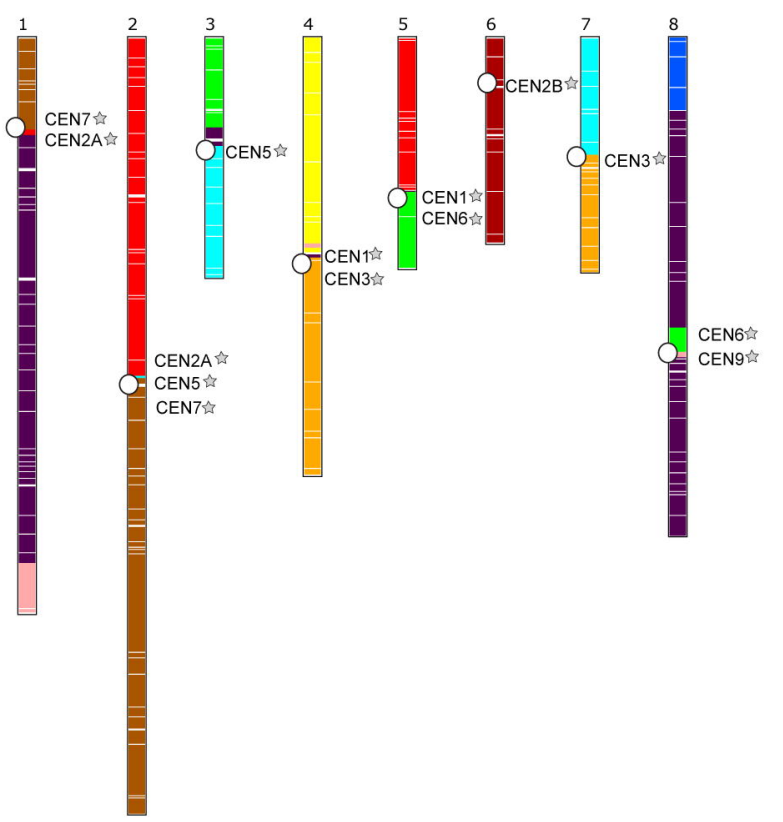




\section{A (CEN1)}

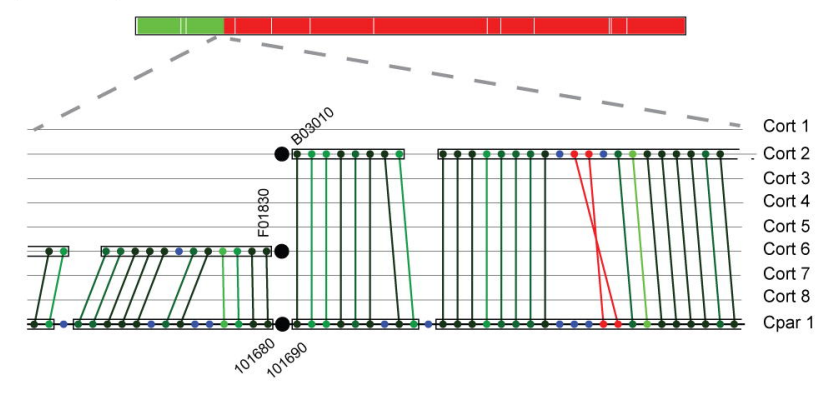

C (CEN3)

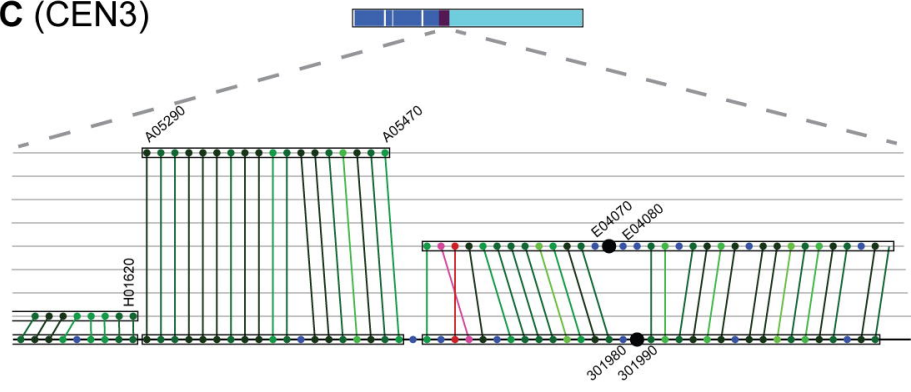

E (CEN5)

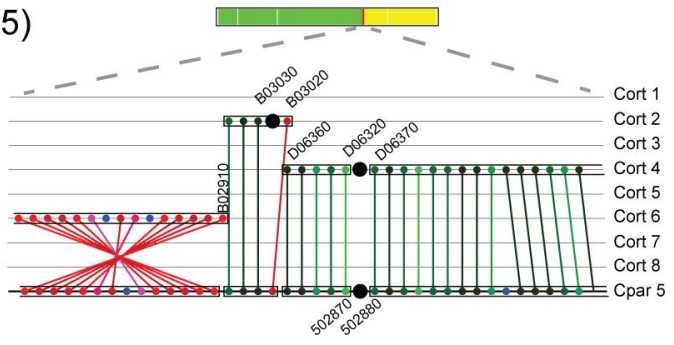

B (CEN2)

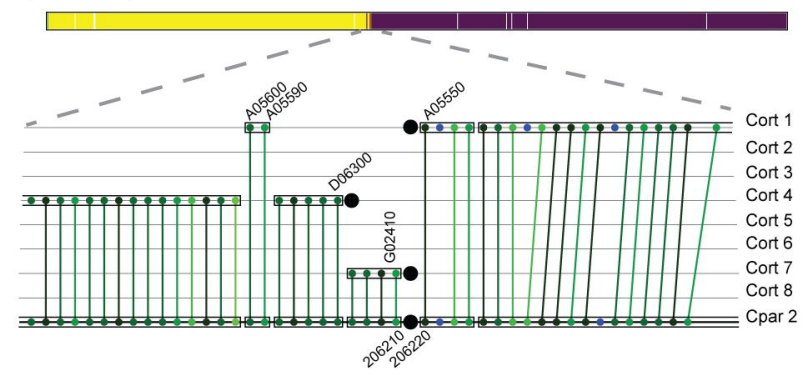

D (CEN4)

Cort 1

Cort 2

Cort 3

Cort 4

Cort 5
Cort 6

Cort 7

Cort 8

Cpar 3

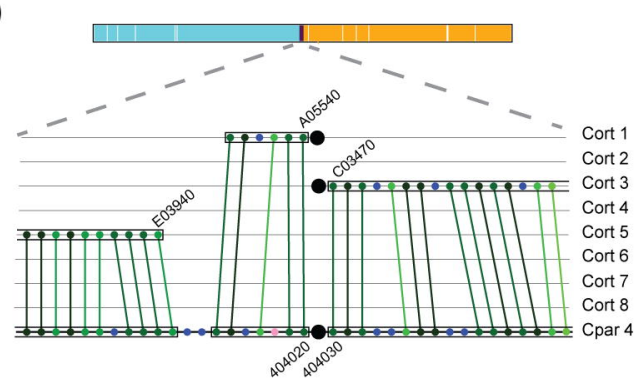

\section{F (CEN6)}

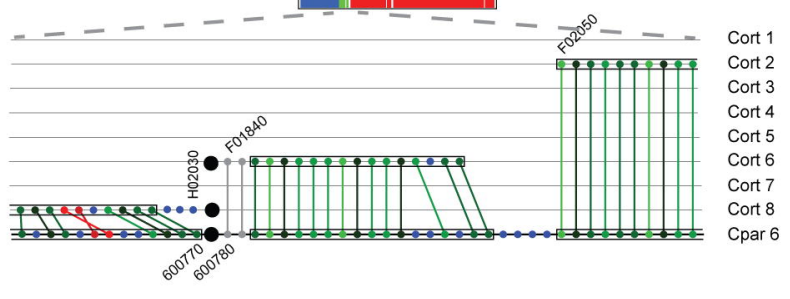

\section{G (CEN7)}

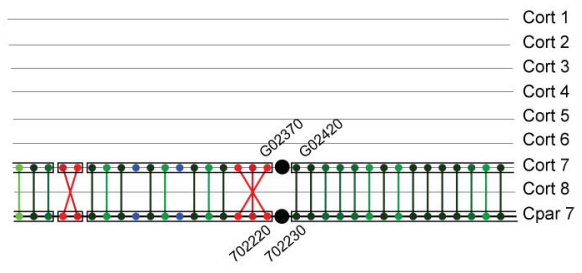

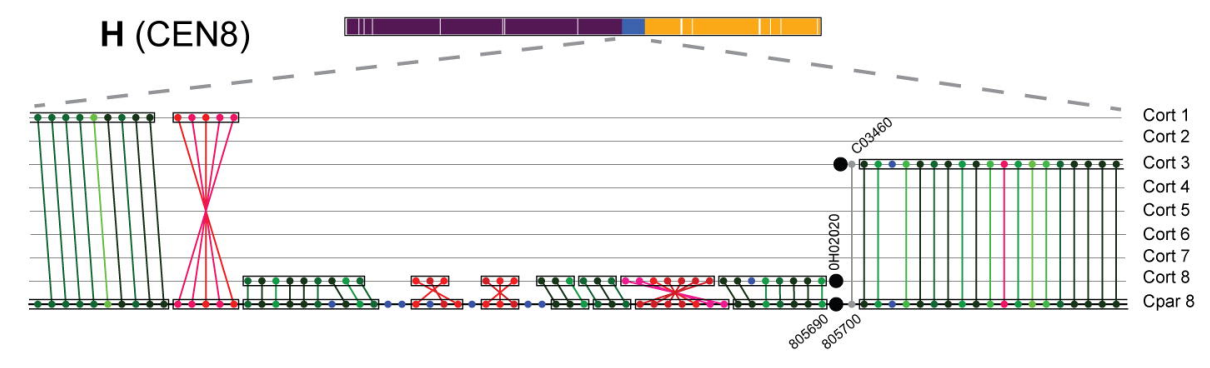


was not certified by peer review) is the author/funder, who has granted bioRxiv a license to display the pr

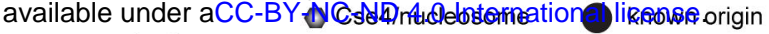

Lipomyces starkeyi

predicted origin

\section{Tortispora starmeri}

Nadsonia fulvescens

Yarrowia lipolytica - - - -

Middelhovenomyces tepae

$?$

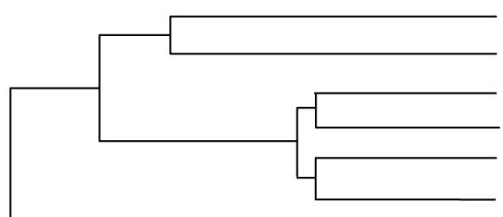

Dipodascus albidus

Candida apicola

Blastobotrys adeninivorans

Alloascoidea hylecoeti

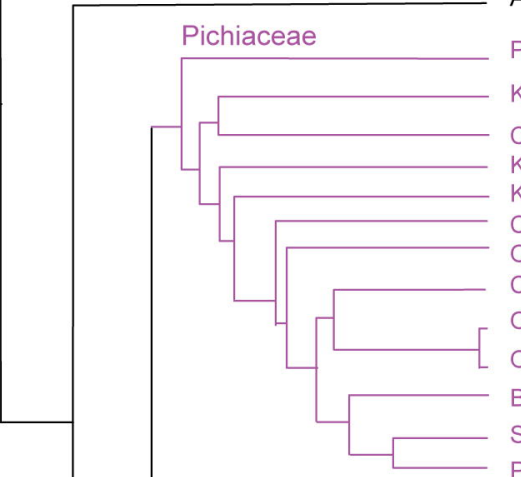

Pachysolen tannophilus

Komagataella pastoris

Citeromyces siamensis

Kuraishia ogatae

Kuraishia capsulata - - - -

Candida boidinii

Ogataea naganishii

Candida arabinofermentans

Ogataea parapolymorpha

Ogataea polymorpha - -

Brettanomyces anomalus

Saturnispora dispora

Pichia kudriavzevii -

Babjeviella inositovora

Cephaloascus fragrans

Kurtzmaniella cleridarum

Meyerozyma guilliermondii

Candida ascalaphidarum

Candida tenuis

Debaryomyces hansenii

Priceomyces haplophilus

Wickerhamia fluorescens

Kodamaea ohmeri

Suhomyces tanzawaensis

Scheffersomyces stipitis

Spathaspora passalidarun

Candida albicans

Candida dubliniensis

Candida tropicalis - _ - -

Lodderomyces elongisporus

Candida parapsilosis

Candida orthopsilosis

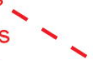

Hyphopichia burtonii

Candida auris

Candida oregonensis

Clavispora lusitaniae -

Metschnikowia bicuspidata -

Saccharomycopsis malanga

Ascoidea rubescens

Candida ponderosae

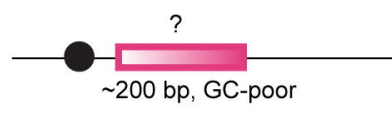

$\sim 200$ bp, GC-poor

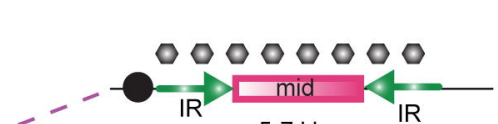

5-7 kb

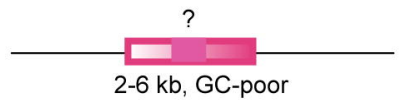

2-6 kb, GC-poor

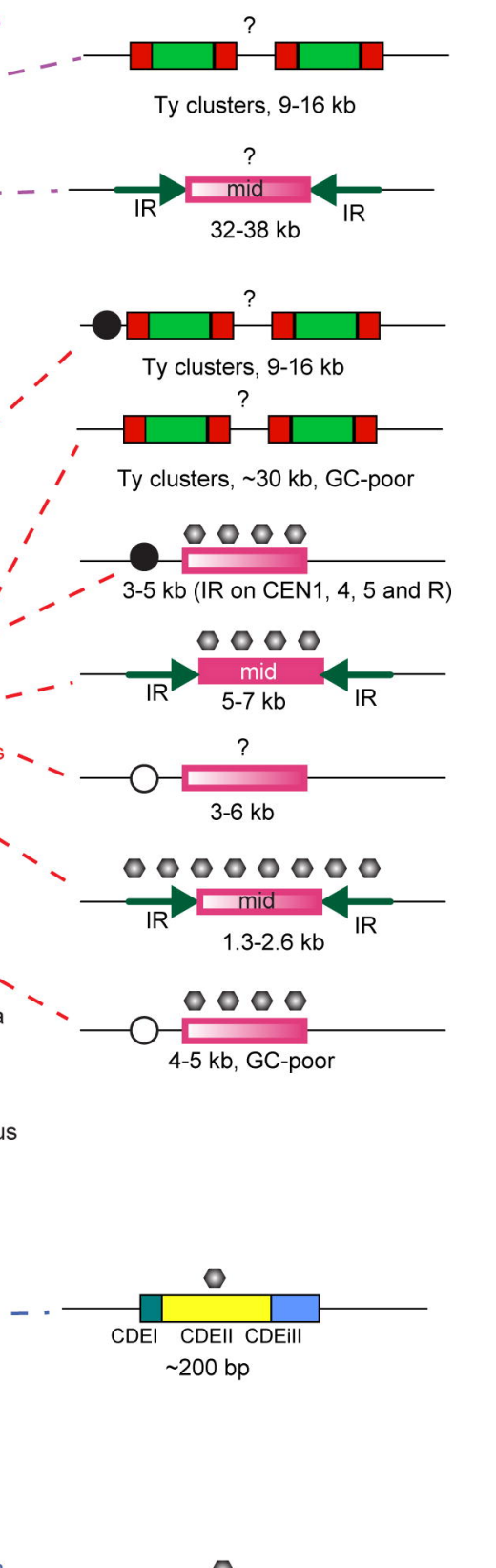

Ty clusters, 9-16 kb
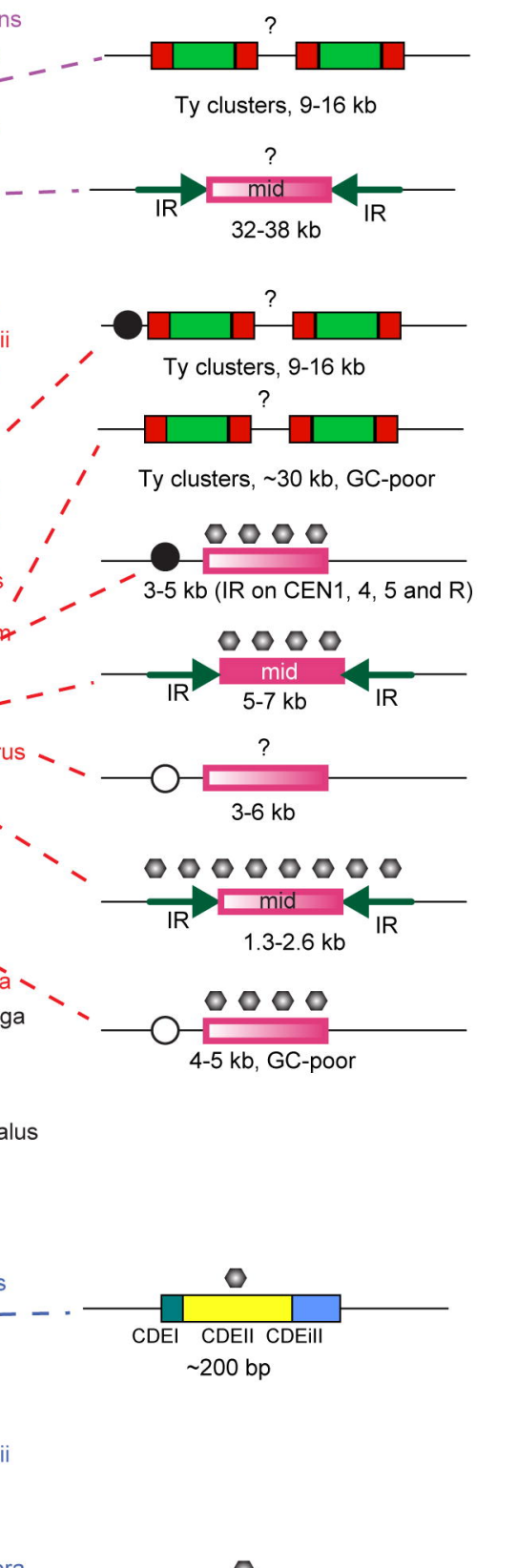

Ty clusters, 9-16 kb

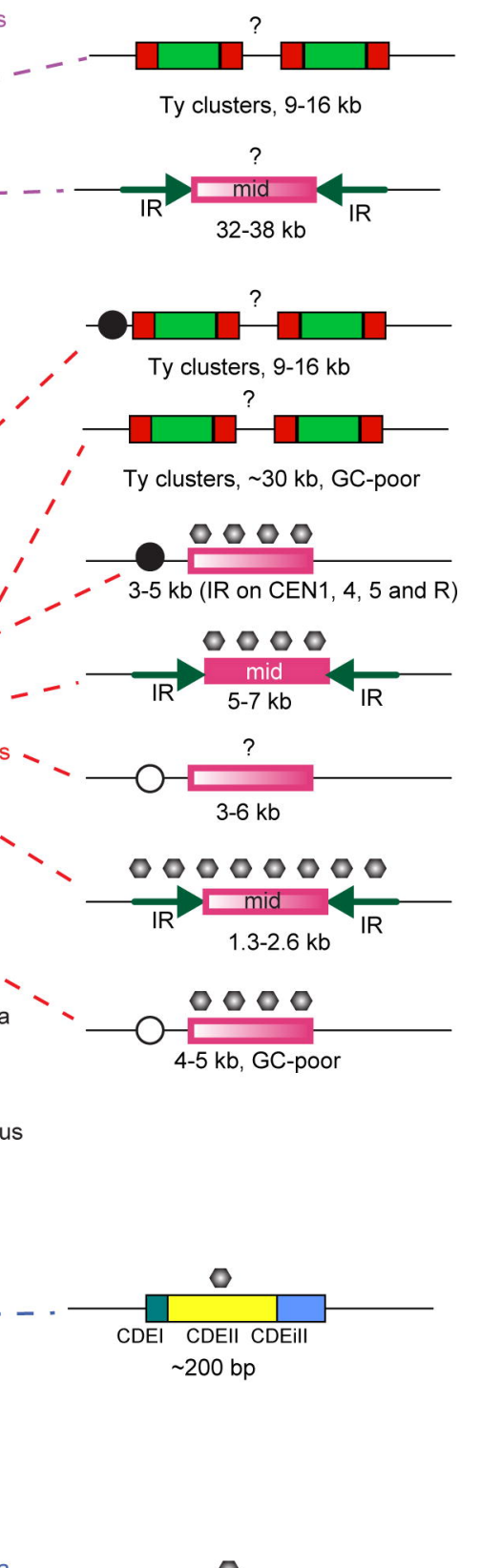

,

Ty clusters, $\sim 30 \mathrm{~kb}, \mathrm{GC}$-poor

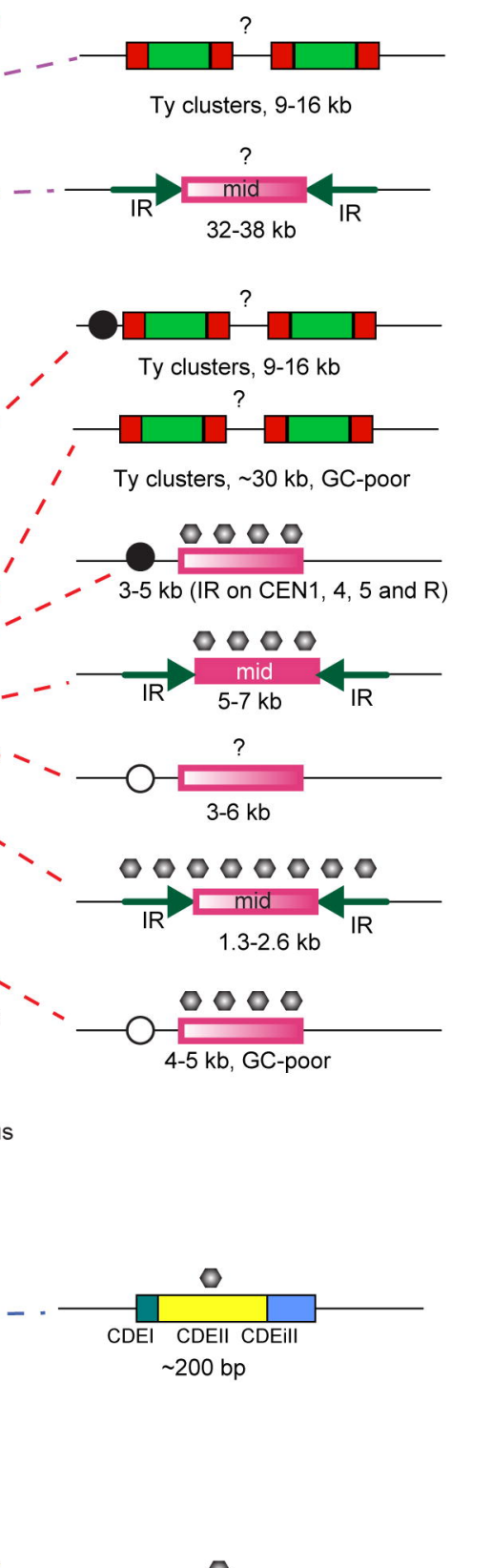

3-5 kb (IR on CEN1, 4, 5 and R) ,
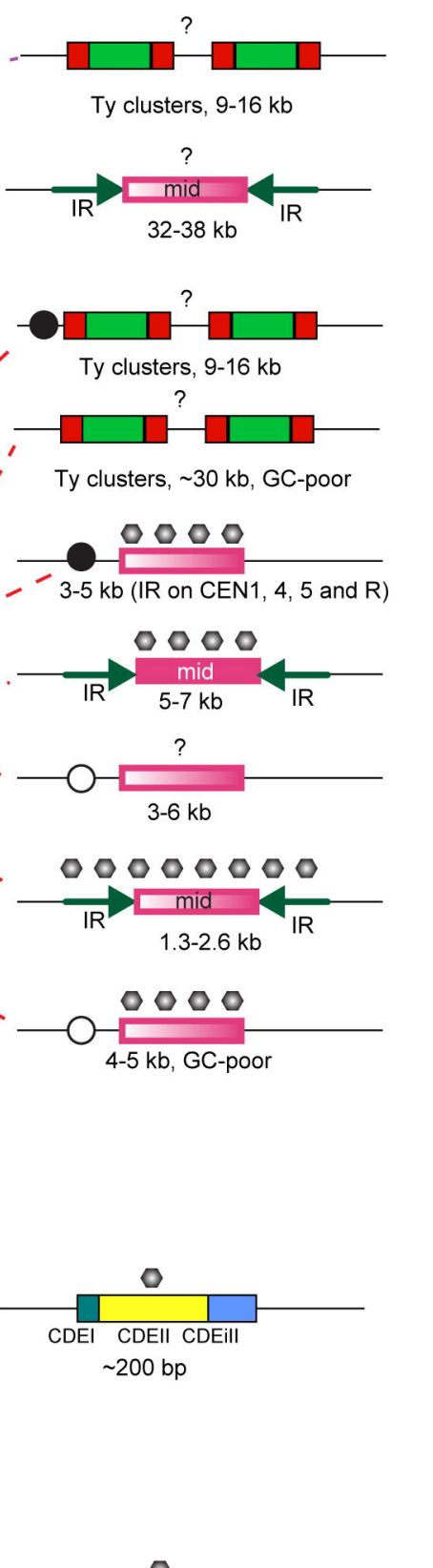

$?$

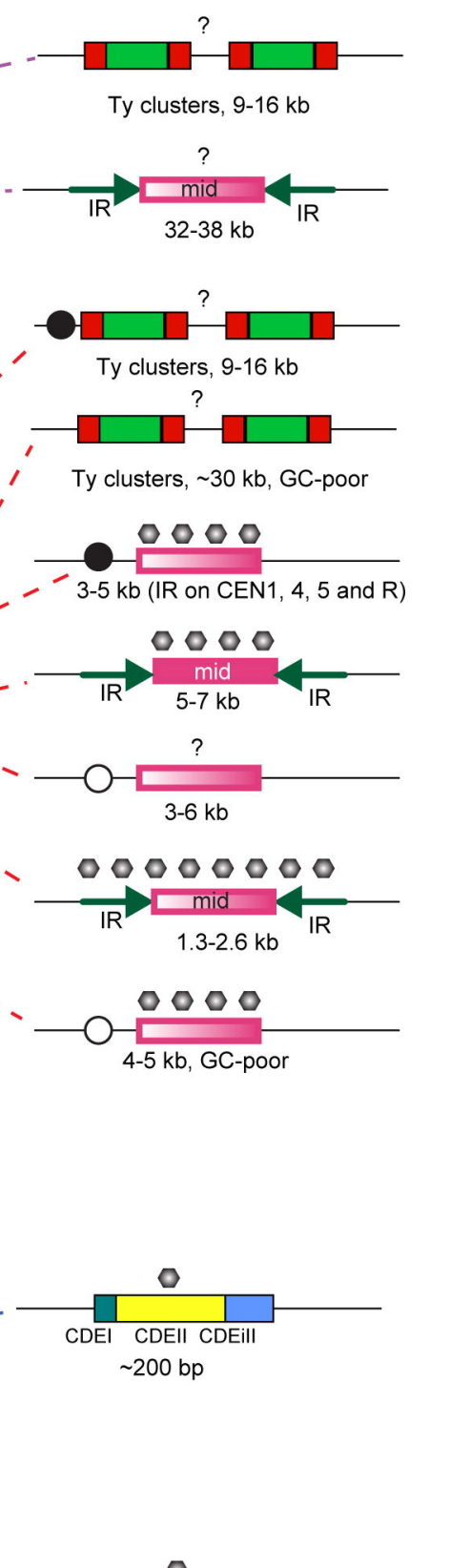

3-6 kb
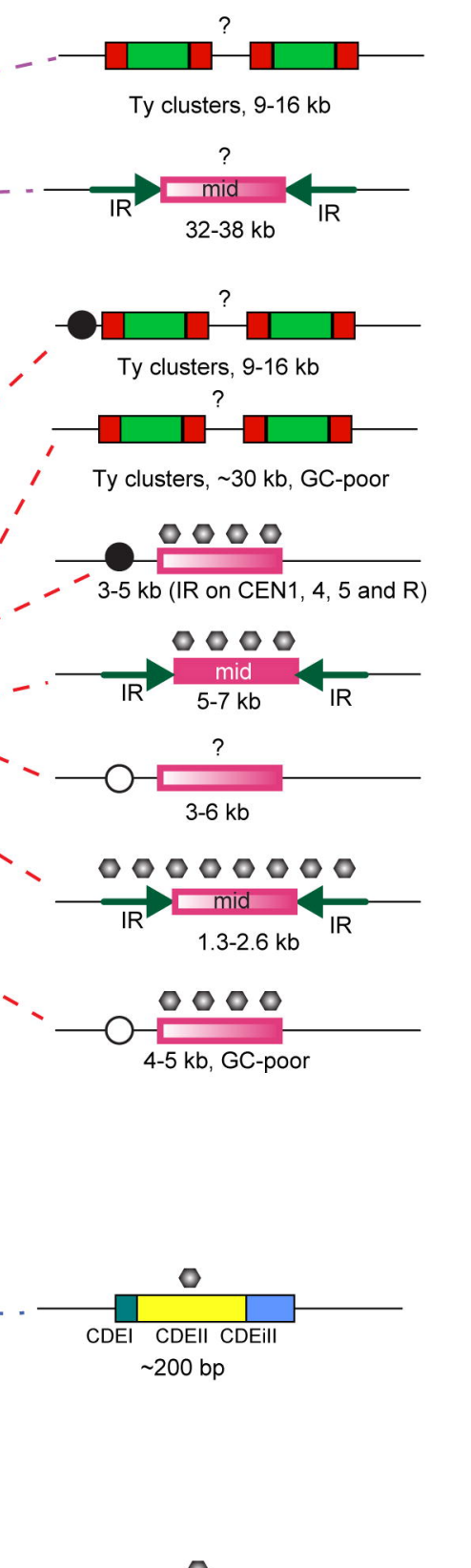

1.3-2.6 kb

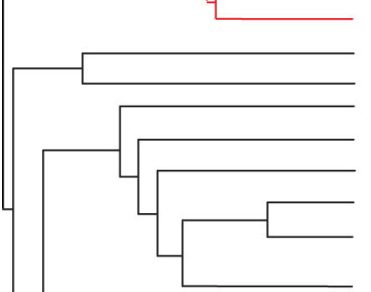

Starmera quercuum

Wickerhamomyces anomalus

Barnettozyma californica

Phaffomyces opuntiae

\section{Cyberlindnera fabianii}

Hanseniaspora valbyensis

Kluyveromyces lactis - - -

0000

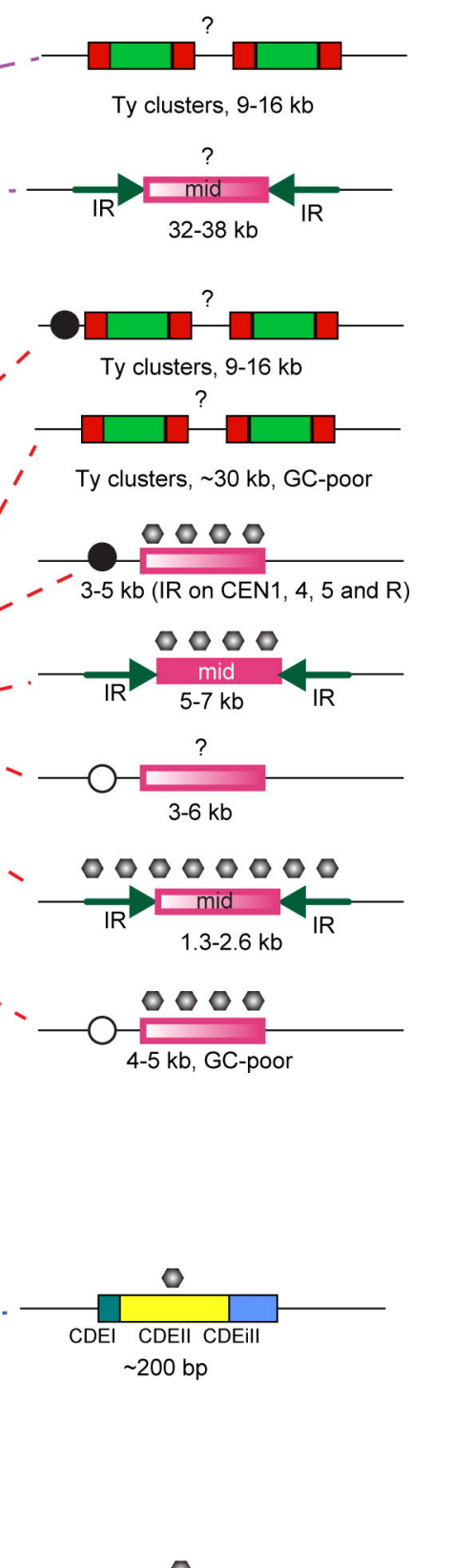

4-5 kb, GC-poor
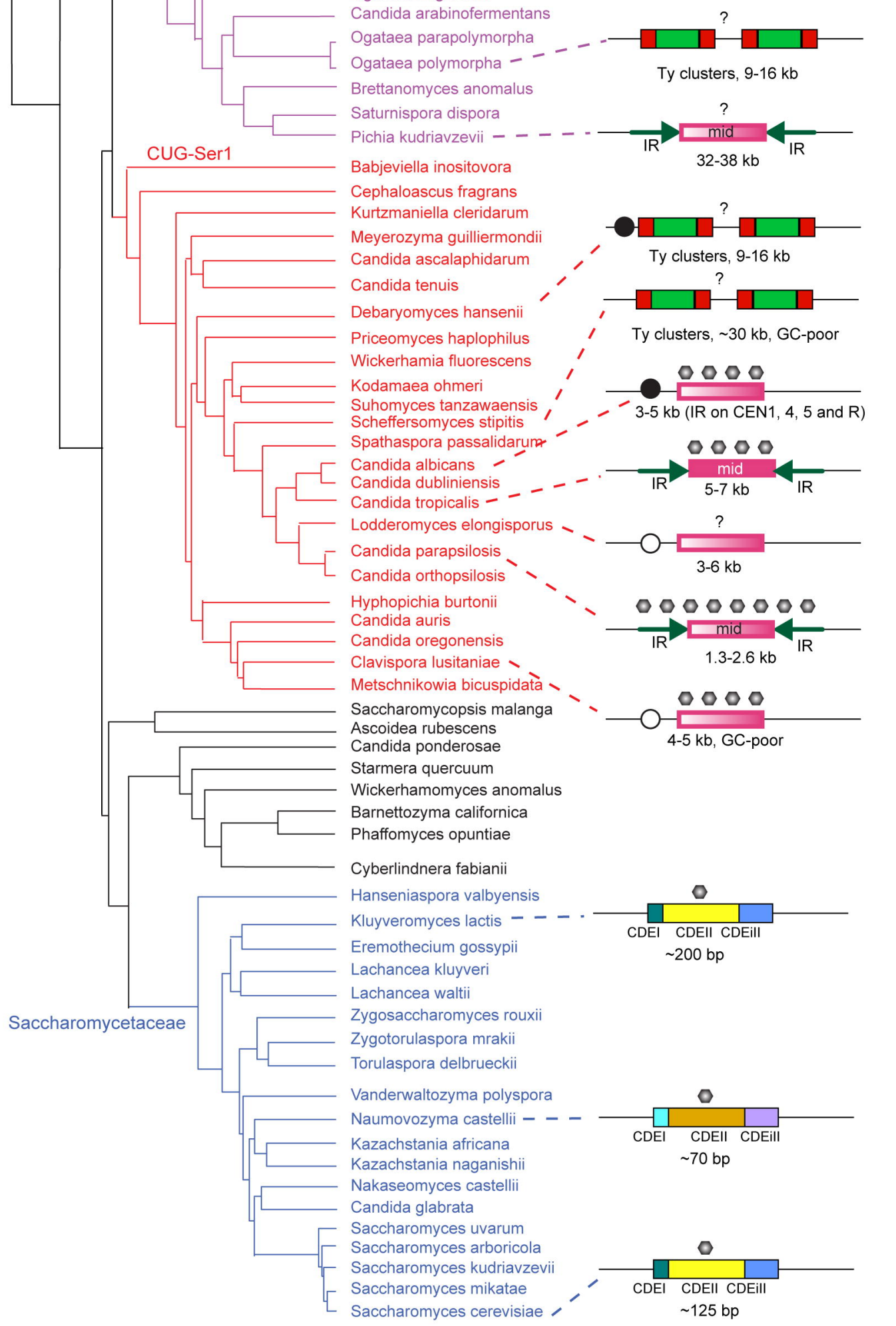

$\sim 200 \mathrm{bp}$

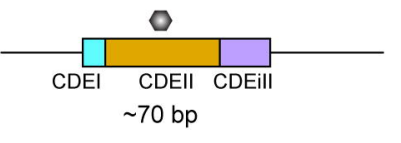

0

CDEI CDEII CDE

$\sim 125 \mathrm{bp}$ 
bioRxiv preprint doi: https://doi.org/10.1101/2020.04.09.034512; this version posted April 10, 2020. The copyright holder for this preprint (which was not certified by peer review) is the author/funder, who has granted bioRxiv a license to display the preprint in perpetuity. It is made

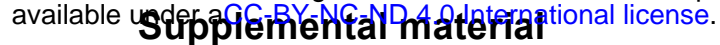

Polymorphic centromere locations in the pathogenic yeast Candida parapsilosis

Supplemental Figure S1 Expression of Cse4-HA and ChIP-PCR

Supplemental Figure S2. Dot matrix plots showing sequence conservation around $C$. orthopsilosis (A) and C. metapsilosis centromeres (B).

Supplemental Figure S3 Rearrangements at putative centromeres in L. elongisporus Supplemental Figure S4. Ancestral reconstruction.

Supplemental Table S1. List of primers used.

Supplemental Table S2. Coordinates of centromeres in C. parapsilosis, C.

orthopsilosis and C. metapsilosis 
bioRxiv preprint doi: https://doi.org/10.1101/2020.04.09.034512; this version posted April 10,2020. The copxright holder for this preprint (which was not certified by peeitreview), is theabithor/funder, who has granted bioRxiv a license to display the preprint in perpetuity. It is made Gly69|Gly70

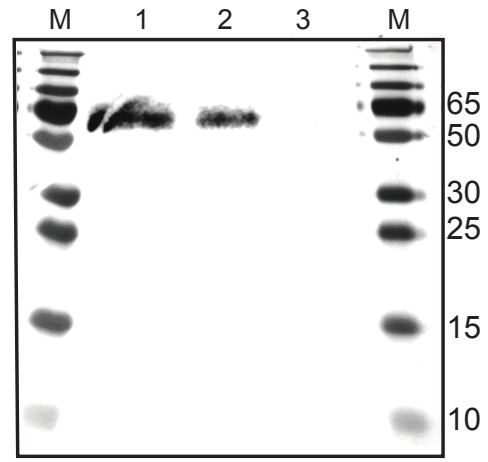

N1

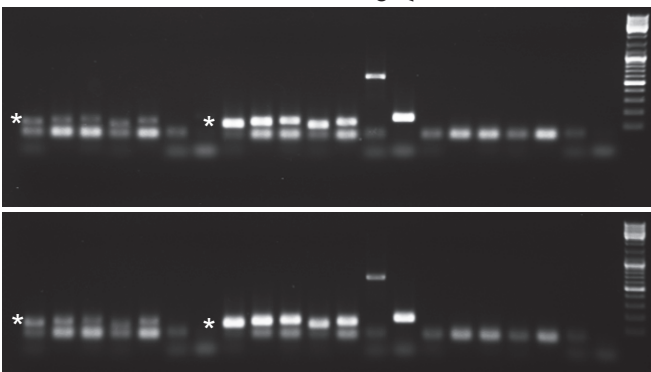

CTRL

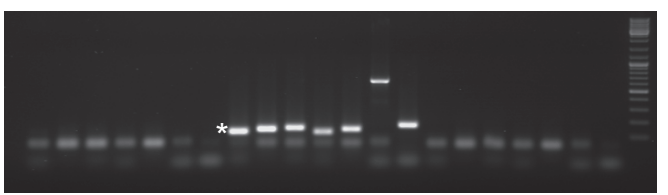

B

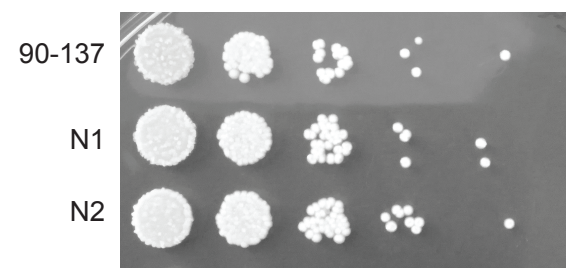

D

Repair template (modified to change PAM and add 3xHA tag)

ACCTCCCCAGTCCTTTCTCATCCCACACATGCAAGCATTGACAAATTATGGCTGATCAACCAACAAATGCCACAAATCCAAGCACTACCAATGCGGCGGGTAACACCACAAGAGGTGTAG GAGAATTAACACCTCAAGAACGCCAACTACAAGAATTGAGAAGACAAAGACAAGAACTAAG GCGTCAACAACAACAACGGGCGCAACAACGTGCCCAACAACAAGGTTCAGCATGGGCAG GTGCAACTGCAGGTTACCCATACGATGTTCCTGACTATGCGGGCTATCCGTATGACGTCCC GGACTATGCAGGATCCTATCCATATGACGTTCCAGATTACGCTGGAGCAGGTTCACCAACA CTAGCTCAATCACCGTTTGCTCGAAGAGCACAAGCAGAAGGTACAGCAACAGGCGCAATG AGATCGCCGTTTCAGTCACCATTTGCTCGAACTAGGGATCAACAACAACAGCGACCTACAC CTGCTCAACCAGAGAGTATACAAAGGATAAATAGAGGAGAGGGATTGGTACCACGCCCGG GTGGTACTGGCGGAGCTGCCAGACCTTCGCCAAGGCCGGGTGCTGATATAG

Supplemental Figure S1 Expression of Cse4-HA and ChIP-PCR

A. Expression of Cse4-HA in C. parapsilosis 90-137 was detected by Western blot using anti-HA antibody. Lane M: PageRuler Plus Prestained Protein ladder (Thermo Scientifics). Sizes are shown in kD. Lanes 1 and 2: protein extract from two independent Cse4-HA tagged derivatives of $C$. parapsilosis 90-137 Lane 3: protein extract from untagged C. parapsilosis 90-137. The protein size is expected to be $49.4 \mathrm{kD}$. B. Tagging Cse4 does not interfere with growth. The tag was introduced at a position that was predicted to be unlikely to interfere with the function of Cse4. Two Cse4-tagged derivatives of C. parapsilosis 90-137 (N1 and N2) grow as well as the parental strain C. parapsilosis 90-137 on YPD. Serial dilutions of cells were spotted on YPD agar and grown for $48 \mathrm{~h}$ at 30 degrees. C. ChIP-PCR from C. parapsilosis CEN1. N1 and N2 are Cse4-tagged derivatives, and CTRL is the untagged strain. PCR amplification was carried out using 5 pairs of primers from within the core region of CEN1 shown in red (Table S1), one pair from an adjacent intergenic region on Chromosome 1 (OUT) and one pair from ACT1. IP = HA immunoprecipitation with anti-HA; INPUT = protein extract before immunoprecipitation; mock IP = no anti-HA antibody used. The target PCR products in the core region are marked with a white asterisk. Some smaller non-specific products were also obtained. All primer pairs from within CEN1 amplified the expected size fragments from total chromatin (Input) from untagged C. parapsilosis 90-137 and from two Cse4-tagged strains. Some non-specific PCR products were also amplified. The CEN1-specific primers also amplified sequences from anti-HA chromatin immunoprecipitates in the tagged strains (N1 and N2), but not in the control untagged strain (CTRL). The OUT and ACT1 primers amplified products from the input samples only. Cse4 therefore localizes to the proposed CEN1. D. Sequence of repair template used to introduce HA tags. 
A C. orthopsilosis centromere region

bioRxiv preprint doi: https://doi.org/10.1101/2020.04.09.034512; this version posted April 10, 2020. The copyright holder for this preprint (which was not certified by peer review) is the author/funder, who has granted bioRxiv a license to display the preprint in perpetuity. It is made

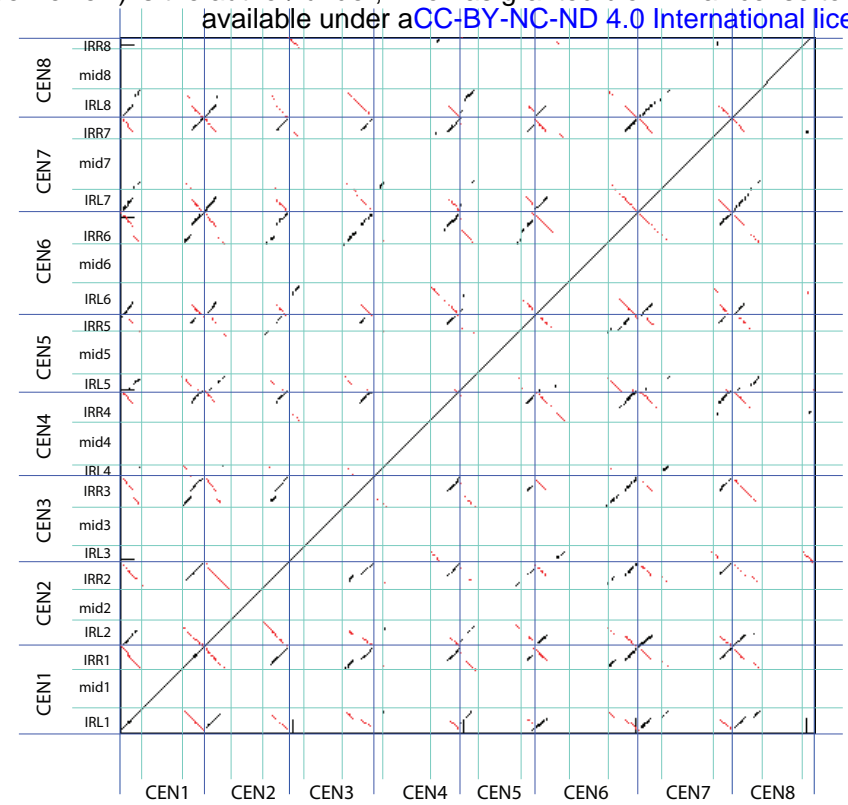

B C. metapsilosis centromere region

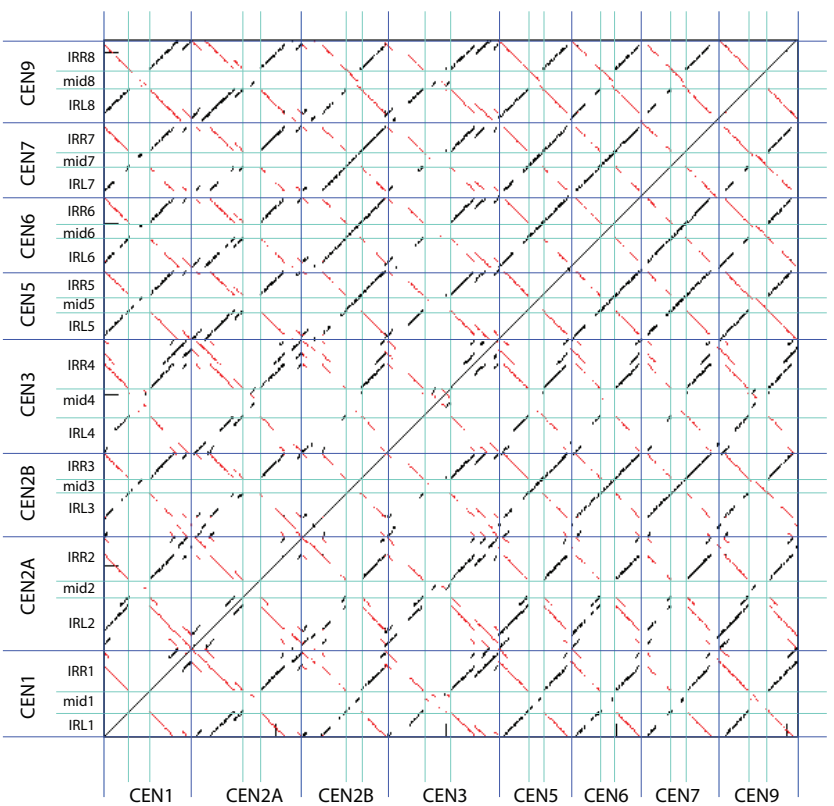

Supplemental Figure S2. Dot matrix plots showing sequence conservation around $C$. orthopsilosis (A) and C. metapsilosis centromeres (B). Centromeres are delineated by dark blue lines. Inverted repeats (Right, IRR and left, IRL) are separated with cyan lines. Each dot represents identity of 25-bp. Inverted sequences are shown in red, and direct repeats in black. 


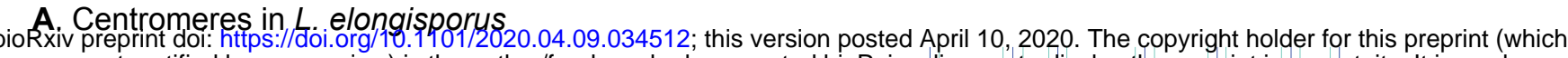
was not certified by peer review) is the author/funder, who has granted bioRxiv a tieense to display the preprint in perpetuity. It is made
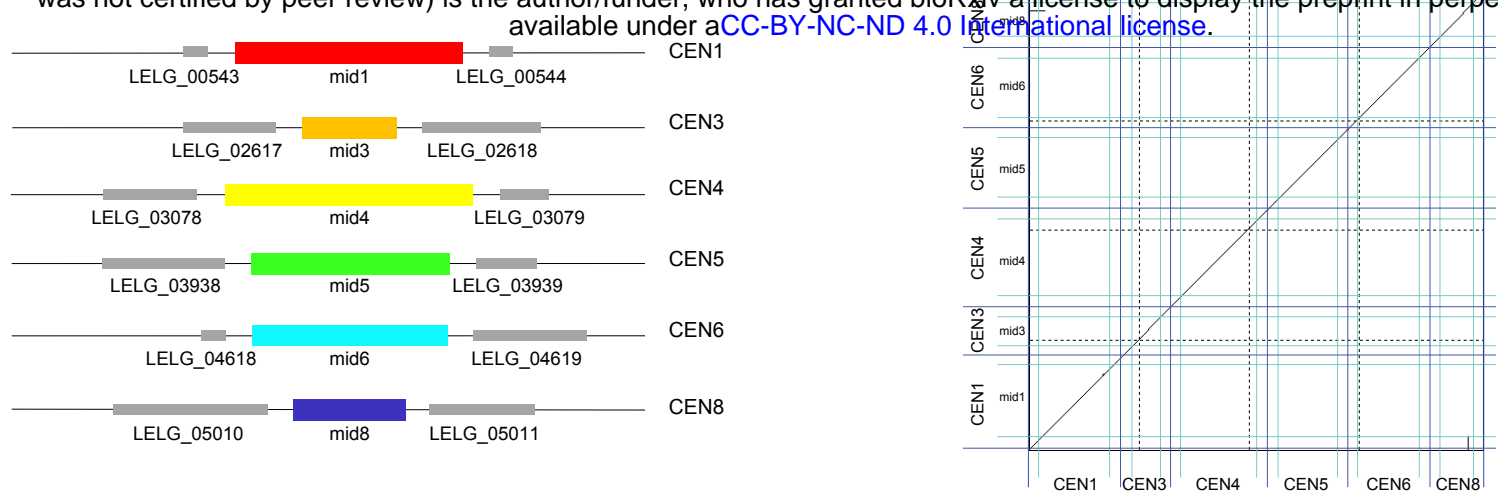

B. L. elongisporus centromeres

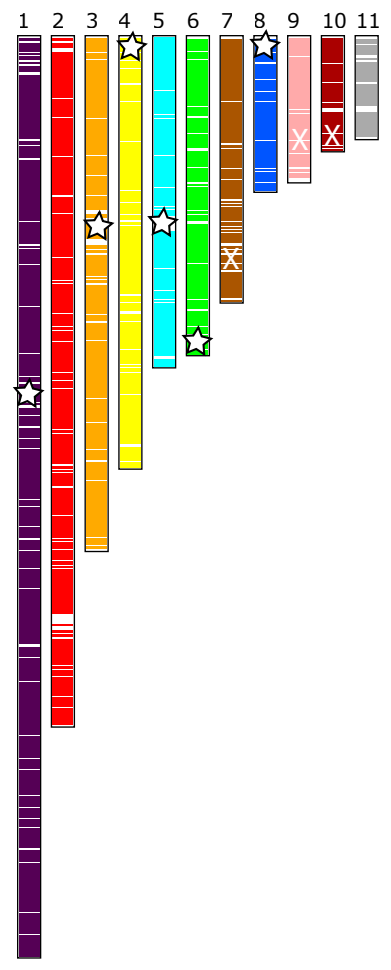

C. C. parapsilosis synteny

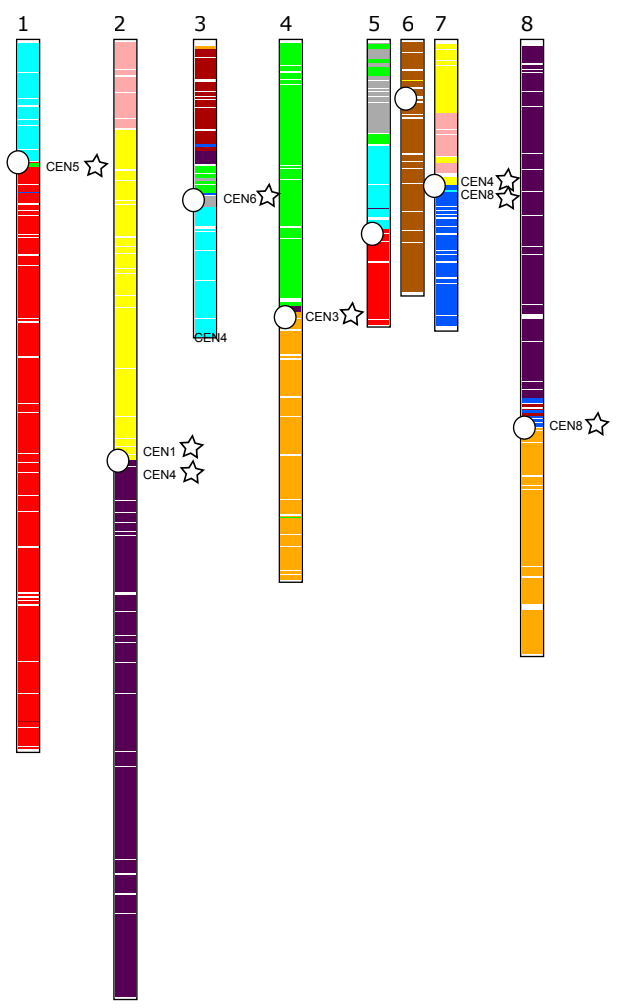

Supplemental Figure S3 Rearrangements at putative centromeres in L. elongisporus

A. Diagrammatic representation of centromeres in L. elongisporus, identified by Koren et al (2010). Only those centromeres that are likely to be correct are shown (i.e those that lie in untranscribed regions (Donovan et al 2016)). The adjacent dotplot shows that there are no repeated sequences around the centromeres.

Synteny relationship between C. parapsilosis and L. elongisporus were identified identified using SynChro. $B$. Location of hits on L. elongisporus chromosomes. The approximate location of the putative $L$. elongisporus centromeres (from A) are indicated with white stars. Three candidates proposed by Koren et al (2010) (marked with white X's) are unlikely to represent centromeres because they are either adjacent to the rDNA (scaffold 9), or on regions that are strongly transcribed (scaffolds 7 and 10).

C. C. parapsilosis chromosomes, colored with respect to the RBHs from L. elongisporus. The location of the putative $C$. parapsilosis centromeres are indicated with a white circle. The location of syntenic $L$. elongisporus centromeres (where identified) are indicated by name and with a white star. 


\section{A.}

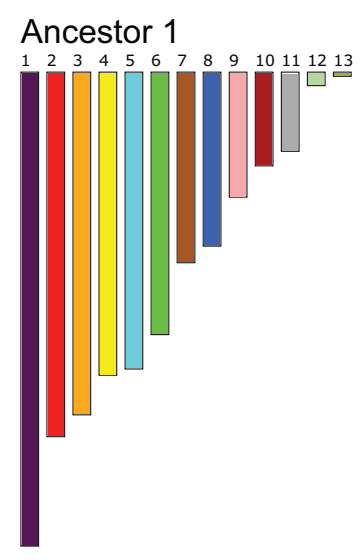

B.

Ancestor 2

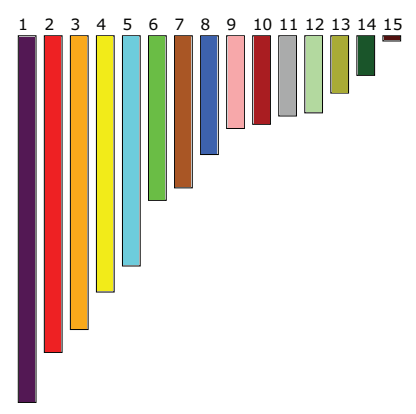

C.

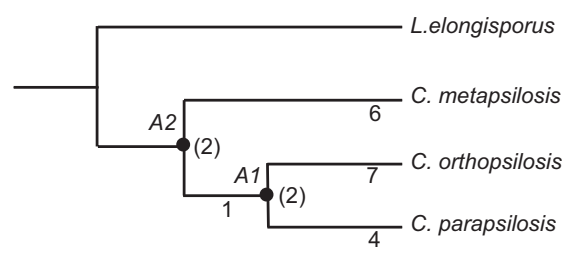

C. parapsilosis

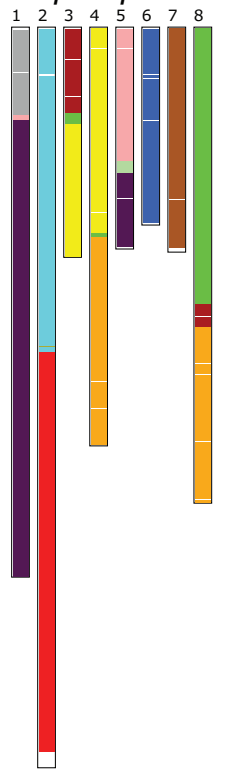

C. orthopsilosis

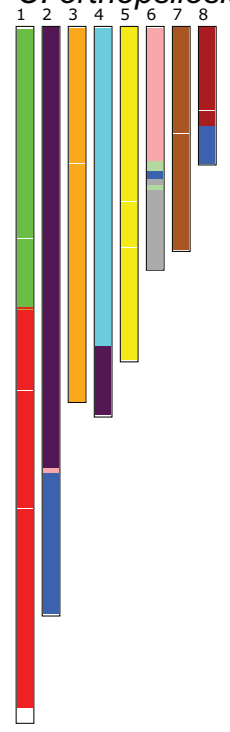

Ancestor 1

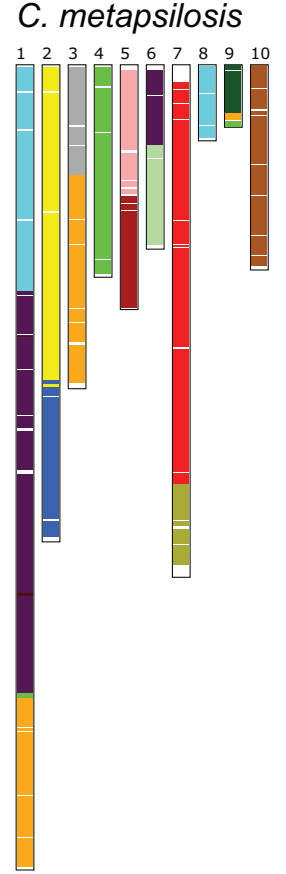

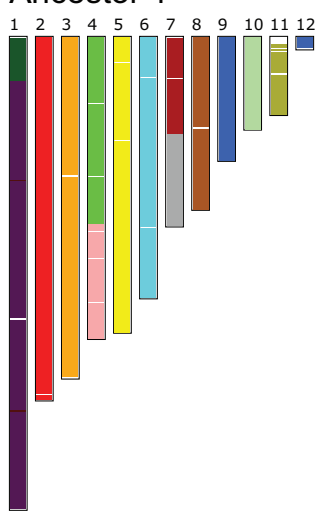

Supplemental Figure S4. Ancestral reconstruction.

A. The genome structure of the ancestor of $C$. parapsilosis and C. orthopsilosis (Ancestor 1, A1) was inferred using AnChro. The sytenic relationship between $\mathrm{A} 1$ and $C$. parapsilosis or $C$. orthopsilosis (determined using SynChro) is shown (see Fig. 4).

B. The genome structure of the ancestor of A1 and C. metapsilosis (Ancestor 2, A2) was inferred as in $(A)$.

C. Interchromosomal breaks (ICBs) were identified by pairwise comparisons of synteny maps from SynChro. Thirteen ICBs were identified between C. parapsilosis and C. orthopsilosis. By comparing with $A 1,7$ were placed on the $C$. orthopsilosis branch, and 4 on the $C$. parapsilosis branch. Two (shown in parantheses) could not be placed. Similar comparisons are shown with Ancestor A2. The phylogeneic relationhip between the species is taken from Pryszcz et al (2015). 
Table S1. Oligonucleotide sequences used in this study.

\begin{tabular}{|c|c|}
\hline Name & Sequence $5^{\prime}-3^{\prime}$ \\
\hline \multicolumn{2}{|c|}{ Guide RNA construction: } \\
\hline gRNA_CSE4_TOP & CCATGGGCAGGTGCAACTGCTGG \\
\hline gRNA_CSE4_BOT & AACCCAGCAGTTGCACCTGCCCA \\
\hline \multicolumn{2}{|l|}{ ChIP-PCR: } \\
\hline \multicolumn{2}{|l|}{ CEN1 } \\
\hline chr1_midCEN1_1_fw & CAAGATGCCCAGAGATGCAG \\
\hline chr1_midCEN1_1_rv & ATCCTACAAGTTCCTACTCG \\
\hline chr1_midCEN1_2_fw & GGGATATTTCGGACAAGTAG \\
\hline chr1_midCEN1_2_rv & CCAAATCAGCAACCAGCAGC \\
\hline chr1_midCEN1_3_fw & GAAGAATTTCGCGTTGACTG \\
\hline chr1_midCEN1_3_rv & CAAATAGTGGTCATACCGTC \\
\hline chr1_midCEN1_4_fw & GCCGCCAACTTAGTTATTAC \\
\hline chr1_midCEN1_4_rv & ATGAACACTTTCTCGGCATG \\
\hline chr1_midCEN1_5_fw & GGATGCAGTAGTATTTGGTG \\
\hline chr1_midCEN1_5_rv & CACCGTTACTGCACCCTTAC \\
\hline \multicolumn{2}{|c|}{ OUT region (chr1:1948277-1955373) } \\
\hline chr1_OUT_fw & TCGGCGCTAGGATCATAACA \\
\hline chr1_OUT_rv & TGCCATCTTGTATTGCACCC \\
\hline \multicolumn{2}{|l|}{ ACT1 } \\
\hline CpACT1_fw & GAAGCTTTGTTCCGTCCAGC \\
\hline CpACT1_rv & TGATGGAGCCAAAGCAGTGA \\
\hline \multicolumn{2}{|c|}{ Colony PCR and Repair Template (RT) amplification: } \\
\hline CSE4_N_RT_fw & ACCTCCCCAGTCCTTTCTCA \\
\hline CSE4_N_RT_rv & TATATCAGCACCCGGCCTTG \\
\hline CSE4_col_inTag_rv & TACGGATAGCCCGCATAGTC \\
\hline
\end{tabular}


bioRxiv preprint doi: https://doi.org/10.1101/2020.04.09.034512; this version posted April 10, 2020. The copyright holder for this preprint (which was not certified by peer review) is the author/funder, who has granted bioRxiv a license to display the preprint in perpetuity. It is made available under aCC-BY-NC-ND 4.0 International license.

\begin{tabular}{|c|c|c|c|c|c|c|c|c|c|c|c|}
\hline Species & IName & Chromosome & Start & End & total length & LR & length LR & $\mathrm{RR}$ & length RR & CORE & length core \\
\hline C.parapsilosis & CENP1 & chr1 & 362074 & 368313 & 26240 & LR_1 & 2827 & $\begin{array}{l}\text { RR_1 } \\
\end{array}$ & 1734 & CORE1 & 1679 \\
\hline \multirow[t]{7}{*}{ CDC317 } & CENP2 & chr2 & 1309230 & 1315533 & 6304 & LR_2 & 2246 & RR_2 & 2248 & CORE2 & 1810 \\
\hline & CENP3 & chr3 & 470791 & 476632 & 5842 & LR_3 & 2052 & $\mathrm{RR}^{-} 3$ & 2489 & CORE3 & 1301 \\
\hline & CENP4 & chr4 & 888739 & 895896 & 7158 & LR_4 & 2291 & RR_4 & 2464 & CORE4 & 2403 \\
\hline & CENP5 & chr5 & 658131 & 664857 & 6727 & LR_5 & 2079 & RR_5 & 1973 & CORE5 & 2675 \\
\hline & CENP6 & chr6 & 209331 & 216041 & 6711 & LR_6 & 2359 & RR_6 & 2259 & CORE6 & 2093 \\
\hline & CENP7 & chr7 & 470717 & 477755 & 7039 & LR_7 & 2916 & RR_7 & 2806 & CORE7 & 1317 \\
\hline & CENP8 & chr8 & 1281311 & 1287676 & 6366 & LR_8 & 2084 & $\mathrm{RR} 8$ & 2383 & CORE8 & 1899 \\
\hline \multirow{8}{*}{\begin{tabular}{|l|} 
C.orthopsilosis \\
90-125 \\
GCA_004334915.1
\end{tabular}} & CENO1 & PQBP01000006.1 & 1221987 & 1227665 & 5679 & LR_1 & 1303 & RR_1 & 1610 & CORE1 & 2766 \\
\hline & CENO2 & PQBP01000001.1 & 1799134 & 1804756 & 5623 & LR_2 & 1581 & RR_2 & 1724 & CORE2 & 2318 \\
\hline & CENO3 & PQBP01000003.1 & 784813 & 790526 & 5714 & LR_3 & 935 & RR_3 & 2012 & CORE3 & 2767 \\
\hline & CENO4 & PQBP01000004.1 & 1287754 & 1293548 & 5795 & LR_4 & 135 & RR_4 & 2207 & CORE4 & 3453 \\
\hline & CENO5 & PQBP01000005.1 & 571630 & 576517 & 4888 & LR_5 & 1031 & RR_5 & 788 & CORE5 & 3069 \\
\hline & CENO6 & PQBP01000007.1 & 653200 & 660294 & 7095 & LR_6 & 2279 & RR_6 & 2209 & CORE6 & 2607 \\
\hline & CENO7 & PQBP01000009.1 & 459407 & 465641 & 6235 & LR_7 & 1300 & RR_7 & 1459 & CORE7 & 3476 \\
\hline & CENO8 & PQBP01000008.1 & 423403 & 428850 & 5448 & LR_8 & 1866 & RR_8 & 936 & CORE8 & 2646 \\
\hline \multirow{8}{*}{$\begin{array}{l}\text { C. metapsilosis } \\
\text { hybrid assembly }\end{array}$} & CENM1 & scaffold1_size3459122 & 978590 & 985894 & 7305 & LR_1 & 2094 & RR_1 & 3581 & CORE1 & 1630 \\
\hline & CENM2A & scaffold2_size2959145 & 1311618 & 1320831 & 9214 & LR_2A & 4308 & RR_2A & 3546 & CORE2A & 1360 \\
\hline & CENM2B & scaffold2_size2959145 & 2207393 & 2214374 & 6982 & LR_2B & 3713 & RR_2B & 2058 & CORE2B & 1211 \\
\hline & CENM3 & scaffold3_size1388033 & 461965 & 471494 & 9530 & LR_3 & 3191 & RR_3 & 4051 & CORE3 & 2288 \\
\hline & CENM5 & scaffold5_size1063617 & 583379 & 589185 & 5807 & LR_5 & 2446 & RR_5 & 2148 & CORE5 & 1213 \\
\hline & CENM6 & scaffold6_size820601 & 335550 & 341602 & 6053 & LR_6 & 2699 & RR_6 & 2142 & CORE6 & 1212 \\
\hline & CENM7 & scaffold7_size2161850 & 1746448 & 1752819 & 6372 & LR_7 & 2582 & RR_7 & 2574 & CORE7 & 1216 \\
\hline & CENM9 & scaffold9_size263853 & 202520 & 209225 & 6706 & LR_9 & 2676 & RR_9 & 2428 & CORE9 & 1602 \\
\hline
\end{tabular}

